\title{
The TyrA family of aromatic-pathway dehydrogenases in phylogenetic context
}

\author{
Jian Song ${ }^{* 1}$, Carol A Bonner ${ }^{2}$, Murray Wolinsky ${ }^{1}$ and Roy A Jensen ${ }^{1,2}$
}

Address: ${ }^{1}$ Los Alamos National Laboratory, Los Alamos, New Mexico, 87545, USA and ${ }^{2}$ Emerson Hall, University of Florida, P.O. Box 14425, Gainesville, Florida, 32604-2425, USA

Email: Jian Song* - jian@lanl.gov; Carol A Bonner - cbonner@ufl.edu; Murray Wolinsky - murray@lanl.gov; Roy A Jensen - rjensen@ufl.edu

* Corresponding author

Published: 12 May 2005

BMC Biology 2005, 3:13 doi:10.1186/1741-7007-3-13
Received: 19 February 2005

Accepted: 12 May 2005

This article is available from: http://www.biomedcentral.com/1741-7007/3/13

(c) 2005 Song et al; licensee BioMed Central Ltd.

This is an Open Access article distributed under the terms of the Creative Commons Attribution License (http://creativecommons.org/licenses/by/2.0), which permits unrestricted use, distribution, and reproduction in any medium, provided the original work is properly cited.

\begin{abstract}
Background: The TyrA protein family includes members that catalyze two dehydrogenase reactions in distinct pathways leading to L-tyrosine and a third reaction that is not part of tyrosine biosynthesis. Family members share a catalytic core region of about $30 \mathrm{kDa}$, where inhibitors operate competitively by acting as substrate mimics. This protein family typifies many that are challenging for bioinformatic analysis because of relatively modest sequence conservation and small size.

Results: Phylogenetic relationships of TyrA domains were evaluated in the context of combinatorial patterns of specificity for the two substrates, as well as the presence or absence of a variety of fusions. An interactive tool is provided for prediction of substrate specificity. Interactive alignments for a suite of catalytic-core TyrA domains of differing specificity are also provided to facilitate phylogenetic analysis. tyrA membership in apparent operons (or supraoperons) was examined, and patterns of conserved synteny in relationship to organismal positions on the $16 \mathrm{~S}$ rRNA tree were ascertained for members of the domain Bacteria. A number of aromatic-pathway genes (his $\mathrm{H}_{b}$, aroF, aroQ) have fused with tyrA, and it must be more than coincidental that the freestanding counterparts of all of the latter fused genes exhibit a distinct trace of syntenic association.

Conclusion: We propose that the ancestral TyrA dehydrogenase had broad specificity for both the cyclohexadienyl and pyridine nucleotide substrates. Indeed, TyrA proteins of this type persist today, but it is also common to find instances of narrowed substrate specificities, as well as of acquisition via gene fusion of additional catalytic domains or regulatory domains. In some clades a qualitative change associated with either narrowed substrate specificity or gene fusion has produced an evolutionary "jump" in the vertical genealogy of TyrA homologs. The evolutionary history of gene organizations that include tyrA can be deduced in genome assemblages of sufficiently close relatives, the most fruitful opportunities currently being in the Proteobacteria. The evolution of TyrA proteins within the broader context of how their regulation evolved and to what extent TyrA co-evolved with other genes as common members of aromatic-pathway regulons is now feasible as an emerging topic of ongoing inquiry.
\end{abstract}




\section{Background}

Dehydrogenases dedicated to L-tyrosine (TYR) biosynthesis comprise a family of TyrA homologs that have different specificities for the cyclohexadienyl substrate: ones specific for L-arogenate (AGN), ones specific for prephenate (PPA), and those that are able to use both $[1,2]$. Figure 1 illustrates the biochemical relationship of these specificities to divergent transformations beginning with chorismate (CHA) utilization and converging on TYR formation. Compounding this complexity, a given TyrA enzyme having any of the aforementioned cyclohexadienyl specificities may be specific for $\mathrm{NAD}^{+}$or $\mathrm{NADP}^{+}$, or may use both. This is consistent with a growing appreciation $[3,4]$ that different substrate specificities are often accommodated across a given protein family that nevertheless maintains a common scaffold of fundamental reaction chemistry. Even within the single category of broad TyrA specificity, there is a continuum ranging from examples where alternative substrates are accepted equally well to other cases where one substrate may be preferred by an order of magnitude or more. Table 1 provides a key to the nomenclature used to identify the various possible substrate-utilization combinations (both cyclohexadienyl and pyridine nucleotide) exhibited by TyrA proteins.

The TyrA family is typical of many protein families in that its members have a relatively small core domain that is not highly conserved. As such, substantial challenges for bioinformatic analysis are posed. Here we have not only carried out a labor-intensive manual analysis, but we have also developed tools intended to facilitate and refine follow-on studies of this protein family in the genome era. The approaches implemented in this study with the TYR segment of aromatic biosynthesis hopefully can serve as a template for forthcoming integrant analyses of other pathway segments of aromatic biosynthesis, and indeed for metabolic subsystems in general.

This manuscript contains three broad sections. First, the biochemical and enzymological complexity of the TyrA protein family is presented in terms of the diversity that exists in nature with respect to substrate specificity and the association of the core domain with other catalytic or regulatory domains. Secondly, the genomic colinear organization of tyrA genes with other genes is evaluated, i.e., tyrA is considered in its syntenic context. Thirdly, tyrA is evaluated in its context of regulation. These three sections are tied together in a framework of evolutionary perspective.

\section{Results and discussion Background of TyrA diversity}

Our evolutionary analysis is limited by the amount of information that can be managed in a single study, with the focus fixed upon the domain Bacteria (due to the rela- tive density of genome representation for Bacteria in the public databases). However, in order to show where future expansion of the analysis might lead, the selection of TyrA proteins in Fig. 2 are from all three domains of life, i.e., Bacteria, Archaea, and Eukarya (lower eukaryotes and higher plants). For practicality of presentation, numerous orphan (i.e., without close relatives) TyrA sequences are not shown, and not all members of a given group are necessarily included. The main purposes of the radial tree shown in Fig. 2 are: (i) to illustrate that TyrA proteins of major phylogenetic groupings are generally congruent with $16 \mathrm{~S}$ rRNA groupings and (ii) to convey a snapshot visualization of the overall complexity of the TyrA protein family from the vantage point of its varied substrate specificities as well as its multiple fusion partners.

As an illustration of the detailed information that follows, note that the TyrA sequences from the beta Proteobacteria at five o'clock in Fig. 2 form a cohesive cluster (termed a 'congruency group'). In this clade there exists a proposed ancestral background of broad specificity where either AGN or PPA in combination with either $\mathrm{NAD}^{+}$or $\mathrm{NADP}^{+}$ could be used. This profile of broad substrate use (which can be denoted as ${ }_{\mathrm{NAD}(\mathrm{P})} \mathrm{TyrA}_{\mathrm{c}^{\prime}}$ see Table 1) generally persists in the beta Proteobacteria. From this background, narrowed specificities for the AGN/NADP+ couple emerged once in the lineage represented by Nitrosomonas europaea (Fig. 2; dark blue line), narrowed specificity for $\mathrm{NAD}^{+}$emerged once in species of Neisseria (orange line), and fusion of $\operatorname{tyr}_{c}$ with aroF (which encodes enolpyruvylshikimate-3-P synthase, the sixth enzyme in the common pathway of aromatic biosynthesis; see $[5,6]$ for nomenclature used) occurred recently within the Burkholderia lineage. These character-state transformations appear to occur with relative ease, and independent emergence of the same character states can be seen elsewhere in the tree.

\section{Phylogenetically congruent TyrA groupings}

Multiple alignments of catalytic-core domains

A phylogenetic tree is only as good as the input alignment. An optimal multiple alignment of TyrA homologs requires a trimmed set of sequences that corresponds to the catalytic-core domain. Alignment of sequences with non-homologous $\mathrm{N}$-terminal fusions (such as with chorismate mutase $\bullet$ (AroQ $\bullet), \mathrm{HisH}_{\mathrm{b}} \bullet$, or plant transit peptides $\bullet$ note the convention of using a bullet to indicate the fusion point of one domain with another domain) will make them appear to be more closely related than they actually are because residues in the non-homologous $\mathrm{N}$-terminal regions find matches at random. Likewise, those TyrA sequences with C-terminal fusions (such as with $\bullet$ AroF, $\bullet$ ACT, or $\bullet$ REG) will appear to be anomalously close to one another. Even enzyme proteins that 

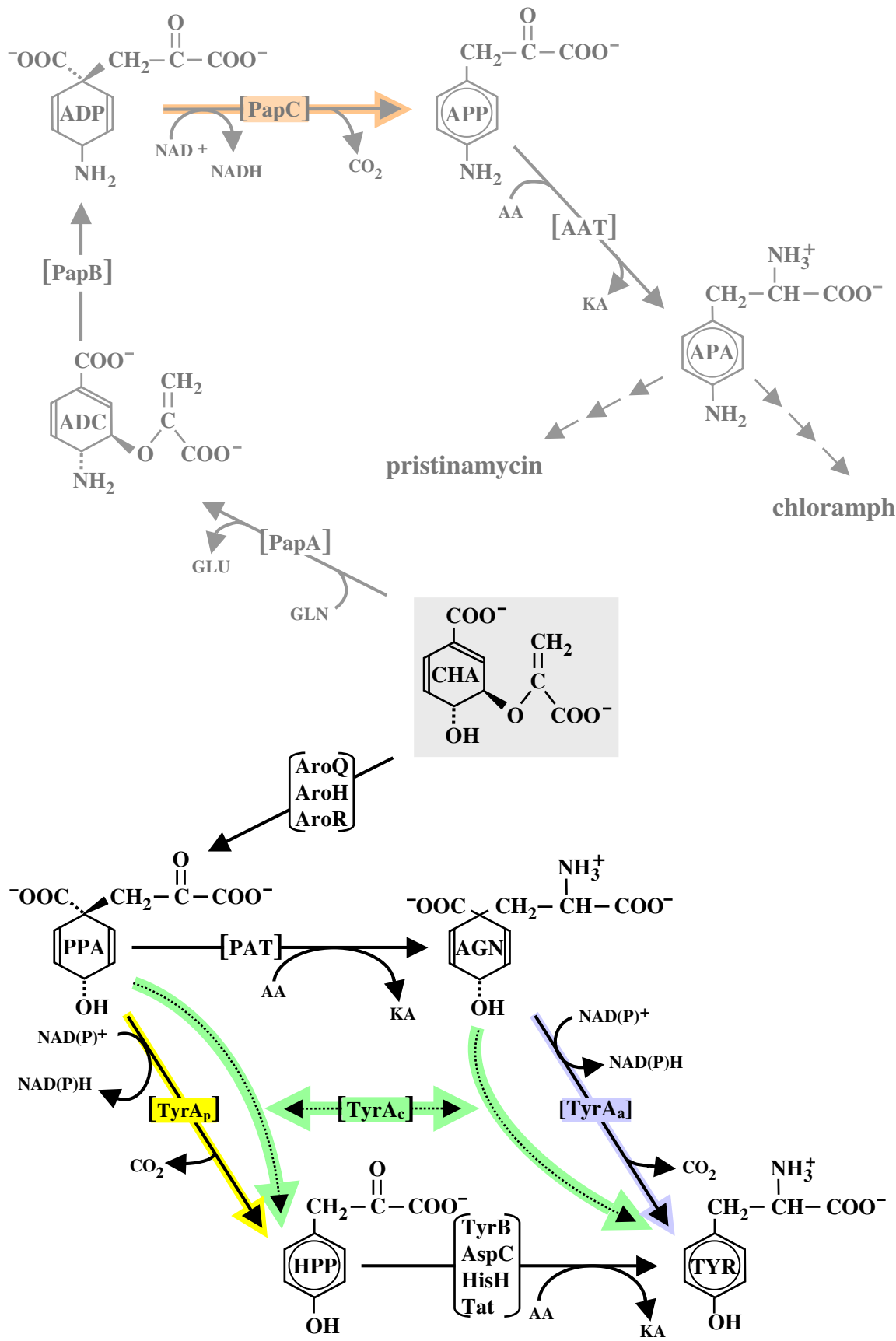

\section{Figure I}

Composite of alternative biochemical routes from chorismate (CHA) to L-tyrosine (TYR) in nature. An antibiotic synthesis branch from $\mathrm{CHA}$ is also shown (dimmed). Here the intermediates shown to intervene between chorismate and pristinamycin or chloramphenicol are $p$-aminochorismate (ADC), $p$-aminoprephenate (ADP), $p$-aminophenylpyruvate (APP), and $p$-aminophenylalanine (APA). PPA may be transaminated by prephenate aminotransferase (PAT) to yield $L$-arogenate (AGN). The four TyrA homologs and the reactions they catalyze are colored differently. Arogenate dehydrogenase $\left(\right.$ TyrA $\left._{\mathrm{a}}\right)$ converts AGN to TYR. Alternatively, prephenate dehydrogenase $\left(\operatorname{TyrA}_{\mathrm{p}}\right)$ converts PPA to 4-hydroxyphenylpyruvate (HPP) which is then transaminated to TYR via an homolog of TyrB, AspC, HisH, or Tat [49]. A broad-specificity cyclohexadienyl dehydrogenase $\left(\operatorname{TyrA}_{c}\right)$ is competent to catalyze either the $\operatorname{TyrA}_{\mathrm{a}}$ or the $\operatorname{TyrA}_{\mathrm{p}}$ reaction. PapC converts the 4-amino analog of PPA to the 4amino analog of HPP. AroQ, AroH, and AroR are distinct homologs known to exist in nature for performance of the chorismate mutase reaction. Other abbreviations: AA, amino acid donor, KA, keto-acid accepter. 
Table I: Abbreviations used to designate substrate specificities of tyrA/TyrA homologs

\begin{tabular}{|c|c|c|}
\hline \multicolumn{2}{|c|}{ Abbreviation $^{\mathrm{a}}$} & \multirow[b]{2}{*}{ Description of specificity ${ }^{b}$} \\
\hline Gene & Gene Product & \\
\hline $\operatorname{tyr}_{\mathrm{x}}$ & TyrA $_{\mathrm{x}}$ & Specificity for cyclohexadienyl substrate is unknown \\
\hline $\operatorname{tyrA}_{\mathrm{c}}$ & TyrA & Broad-specificity cyclohexadienyl dehydrogenase (CDH) \\
\hline$t_{y r} A_{p}$ & TyrA & Narrow-specificity prephenate dehydrogenase $(\mathrm{PDH})$ \\
\hline $\mathrm{TyrA}_{\mathrm{c} \_\Delta}$ & $\operatorname{TyrA}_{\mathrm{c} \_\Delta}$ & Broad-specificity cyclohexadienyl dehydrogenase having catalytic-core indels in correlation with an extra-core extension \\
\hline $\operatorname{tyrA}_{\mathrm{a}}{ }_{\mathrm{c}} \Delta$ & $\operatorname{TyrA}_{\mathrm{a}} \mathrm{C}^{\prime}$ & Narrow-specificity arogenate dehydrogenase (ADH) \\
\hline NADtyrA & NADTyrA & TyrA homolog is AGN-specific and NAD ${ }^{+}$-specific \\
\hline $\begin{array}{l}\text { NADP } t y r A \\
\text { a }\end{array}$ & NADPTyrA & TyrA homolog is AGN-specific and $\mathrm{NADP}^{+}$-specific \\
\hline $\begin{array}{l}\text { NAD }(P) t y r \\
A_{a}\end{array}$ & $\operatorname{NAD}(P) T^{T y r} A_{a}$ & TyrA homolog is AGN-specific but utilizes either $\mathrm{NAD}^{+}$or $\mathrm{NADP}^{+}$ \\
\hline$x^{t y r A} A_{x}$ & $x^{T y r A} A_{x}$ & Specificity for both the cyclohexadienyl and pyridine nucleotide substrates is unknown \\
\hline
\end{tabular}

aAbbreviations in the upper-table (upper 5 rows) indicate the specificities for the cyclohexadienyl substrate. Abbreviations in the lower-table (lower 4 rows) indicate specificities for both cyclohexadienyl (right subscripts) and pyridine nucleotide substrates (left subscripts). Combinations not shown can be deduced from the examples given, e.g., a TyrA homolog specific for prephenate and NAD+ would be designated ${ }_{N A D} T_{y r A}$.

bThe abbreviations $\mathrm{CDH}, \mathrm{PDH}$, and $\mathrm{ADH}$ (shown parenthetically) have been used frequently in the literature.

have much greater sequence conservation and amino-acid lengths than TyrA proteins cannot reasonably be expected to yield a protein tree that would be congruent over an extensive phylogenetic range with the overall 16S rRNA tree. However, if genome representation is sufficiently dense within a range of closely related organisms, 16S rRNA congruency with a given protein can be expected within that range of organisms provided that (i) the particular functional role has been retained and (ii) lateral gene transfer has not occurred to obscure the relationship. This expectation follows from the outcome of a detailed analysis of tryptophan-pathway proteins in Bacteria $[7,8]$.

\section{Congruency within major clades}

TyrA sequences from higher-plant and yeast Eukarya form cohesive clusters. Genome representation among Archaea is still relatively limited. (Fig. 2 does reveal, however, that genes encoding TyrA proteins in Archaea have experienced various catalytic- and regulatory-domain fusions at least as frequently as those in Bacteria). Eventual expansion of both the tryptophan-pathway and tyrosine-pathway analyses to Archaea should be quite interesting.

The great majority of TyrA sequences available are from Bacteria, and one can see (by inspection of the major clades supported by high bootstrap values in Fig. 2) a qualitatively apparent congruence of TyrA-tree sub-sections with 16S rRNA expectations of vertical genealogy. Thus, all cyanobacteria possess a ${ }_{\text {NADP }}$ TyrA $\mathrm{a}_{\mathrm{a}}$ type of TyrA enzyme, and this is a very cohesive grouping. A few of the larger cyanobacterial genomes have a co-existing second enzyme of the TyrA $A_{c \Delta}$ type (discussed in detail later). The low-GC gram-positive bacteria (Bacillus/Staphylococcus/ Enterococcus/Listeria) exhibit the ${ }_{\mathrm{NAD}} \mathrm{TyrA}_{\mathrm{p}}$ pattern of spe- cificity and also possess a C-terminal domain (ACT) of allosteric regulation. It is interesting that the $\operatorname{TyrA}_{\mathrm{p}} \bullet \mathrm{ACT}$ proteins of the Streptococcus lineage (at eight o'clock in Fig. 2) differ from the main low-GC clade in possessing broad specificity for pyridine nucleotides (as indicated with black line color). The most parsimonious evolutionary conclusion would be that in the low-GC gram-positive grouping, acquisition of the ACT domain and narrowed specificity for prephenate preceded narrowed specificity for $\mathrm{NAD}^{+}$. Thus, the latter event occurred after divergence of the Streptococcus lineage from the remainder of the lowGC clade. Members of the subclass taxon Actinobacteridae (mostly actinomycetes) possess AGN-specific TyrA enzymes (light blue fill color in Fig. 2), but they separate into two distinct groups that correlate either with broad specificity for pyridine nucleotides (Actinobacteridae_1) or a NAD ${ }^{+}$-specific pattern (Actinobacteridae_2). The Proteobacteria are discussed immediately below.

\section{Proteobacteria}

By far the greatest genomic density available is for Proteobacteria, the group of Bacteria that includes purple bacteria and their relatives. The various divisions of Proteobacteria, as currently named, lack hierarchical equivalence. For example, the epsilon and delta divisions branch from much deeper positions on the phylogenetic tree than do the alpha Proteobacteria. As genome representation expands for epsilon and delta Proteobacteria, it is probable that these will subdivide to newly named groupings of approximate hierarchical equivalence with alpha Proteobacteria. The most recently diverged Proteobacteria are the beta and gamma divisions. From the combination of our previous analysis of tryptophan biosynthesis [7,8], TYR biosynthesis (this paper), and 
Table 2: Key to organism acronyms

\begin{tabular}{|c|c|c|}
\hline Organism & $\begin{array}{l}\text { Abbreviation } \\
\text { in Paper }\end{array}$ & $\begin{array}{l}\text { Abbreviation } \\
\text { on website }^{b}\end{array}$ \\
\hline Acidithiobacillus ferrooxidans ATCC 23270 & Aferr & \\
\hline Acinetobacter sp. ADPI & $\mathrm{ACIN}$ & \\
\hline Actinobacillus actinomycetemcomitans HK I65I & Aact & \\
\hline Actinomyces naeslundii MGI & Anae & \\
\hline Actinoplanes teichomyceticus & Atei & \\
\hline Agrobacterium tumefaciens strain C58 & Atum & \\
\hline Amycolatopsis balhimycina & Abal & \\
\hline Amycolatopsis orientalis & Aori & \\
\hline Anabaena sp. PCC 7120 & $A N A B$ & \\
\hline Arabidopsis thaliana & Atha & \\
\hline Archaeoglobus fulgidus DSM 4304 & Aful & Aful_l \\
\hline Azotobacter vinelandii & Avin & Avin_l \\
\hline Bacillis anthracis str. A2012 & Bant & \\
\hline Bacillus cereus ATCC I4579 & Bcer & \\
\hline Bacillus halodurans C-125 & Bhal & Bhal_2 \\
\hline Bacillus stearothermophilus & Bste & \\
\hline Bacillis subtilis & Bsub & \\
\hline Bacillus thuringiensis israelensis & Bthu & \\
\hline Bifidobacterium longum NCC2705 & Blon & Blon_I \\
\hline Blochmannia floridanus & Bflo & \\
\hline Bordetella bronchisepticus & Bbro & \\
\hline Burkholderia cepacia J23।5 & Bcep & \\
\hline Burkholderia fungorum LB400 & Bfun & \\
\hline Burkholderia mallei ATCC 23344 & Bmal & \\
\hline Burkholderia pseudomallei K96243 & Bpse & Bpse_6 \\
\hline Campylobacter jejuni & Cjej & \\
\hline Chromobacterium violaceum ATCC 12472 & Cvio & \\
\hline Corynebacterium diphtheriae NCTC 13129 & Cdip & \\
\hline Corynebacterium efficiens YS-314 & Ceff & \\
\hline Corynebacterium glutamicum ATCC 13032 & Cglu & Cglu_I \\
\hline Desulfovibrio desulfuricans $\mathrm{G} 20$ & Ddes & \\
\hline Desulfovibrio vulgaris subsp. vulgaris strain Hildenborough & Dvul & \\
\hline Desulfuromonas acetoxidans & Dace & Dace_5 \\
\hline Enterococcus faecalis V583 & Efae_2 & \\
\hline Enterococcus faecium & Efae_l & Efae_I \\
\hline Erwinia carotovoa subsp.atroseptica SCRII043 & Ecar & \\
\hline Escherichia coli K12 & Ecol & \\
\hline Geobacter metallireducens GS-15 & Gmet & \\
\hline Geobacter sulfurreducens PCA & Gsul & \\
\hline Gloeobacter violaceus PCC 742I & Gvio & \\
\hline Haemophilus influenzae Rd KW20 & Hinf & \\
\hline Helicobacter hepaticus ATCC 5I449 & Hhep & \\
\hline Helicobacter pylori 26695 & Hpyl & \\
\hline Klebsiella pneumoniae subsp. pneumoniae MGH 78578 & Kpne & \\
\hline Leifsonia xyli subsp. Xyli strain СТСВ07 & Lxyl & \\
\hline Listeria innocua Clip I I 262 & Linn & \\
\hline Listeria monocytogenes EGD-e & Lmon & \\
\hline Lotus corniculatus var. japonicus & Lcor & Lcor_3 \\
\hline Lycopersicon esculentum & Lesc & \\
\hline Methanococcus jannaschii & Mjan & \\
\hline Methanopyrus kandleri AVI9 & Mkan & Mkan_l \\
\hline Methanosarcina barkeri strain Fusaro & Mbar & \\
\hline Methanothermobacter thermoautotrophicus strain Delta $\mathrm{H}$ & Mthe & Mthe_7 \\
\hline Microbulbifer degradans $2-40$ & Mdeg & \\
\hline Mycobacterium avium subsp. paratuberculosis strain $\mathrm{k} 10$ & Mavi & \\
\hline Mycobacterium bovis TrEMBL & Mbov & Mbov_2 \\
\hline Mycobacterium leprae TN & Mlep & \\
\hline Mycobacterium tuberculosis CDCI55I & Mtub & \\
\hline Myxococcus xanthus DK 1622 & Mxan & \\
\hline
\end{tabular}


Table 2: Key to organism acronyms (Continued)

\begin{tabular}{|c|c|c|}
\hline Neisseria gonorrhoeae FA 1090 & Ngon & \\
\hline Nitrosomonas europaea ATCC 19718 & Neur & \\
\hline Nocardia farcinica IFM 10152 & Nfar & \\
\hline Nonomuraea sp. & NONO & \\
\hline Nostoc punctiforme PCC73102 & Npun & Npun_I \\
\hline Novosphingomonas aromaticivorans DSM I2444 & Naro & \\
\hline Oceanobacillus iheyensis THE83। & Oihe & \\
\hline Oryza sativa ssp. japonica & Osat & \\
\hline Pantoea agglomerans & Pagg & \\
\hline Pasteurella multocida subsp. multocida strain Pm70 & Pmul & \\
\hline Photorhabdus luminescens subsp. laumondii TTOI & Plum & \\
\hline Prochlorococcus marinus subsp. pastoris strain CCMPI378 & Pmar_I & Pmar_3 \\
\hline Prochlorococcus marinus MIT93I3 & Pmar_2 & Pmar_10 \\
\hline Propionibacterium acnes KPAI7/202 & Pacn & \\
\hline Pseudomonas aeruginosa PAOI & Paer & Paer_I \\
\hline Pseudomonas fluorescens PfO-I & Pflu & \\
\hline Pseudomonas putida KT2440 & Pput & \\
\hline Pseudomonas stutzeri & Pstu & \\
\hline Ralstonia eutropha JMPI34 & Reut & \\
\hline Ralstonia solanacearum GMII 000 & Rsol & \\
\hline Rhodobacter capsulatus & Rcap & \\
\hline Rhodobacter sphaeroides 2.4.I & Rsph & \\
\hline Rhodopseudomonas palustris CGA009 & Rpal & \\
\hline Rhodospirillum rubrum & Rrub & Rrub_I \\
\hline Rubrobacter xylanophilus DSM 994I & Rxyl & \\
\hline Saccharomyces cerevisiae & Scer & \\
\hline Salmonella typhimurium LT2 & Styp & Styp_I \\
\hline Schizosaccharomyces pombe & Spom & \\
\hline Shewanella oneidensis MR-I & Sone & \\
\hline Shewanella putrifacians & Spu & \\
\hline Staphylococcus aureus subsp. Aureus MW2 & Saur & Saur_2 \\
\hline Streptococcus gordonii str. Challis & Sgor & \\
\hline Streptococcus pneumoniae R6 & Spne & \\
\hline Streptomyces avermitilis MA-4680 & Save & \\
\hline Streptomyces caeruleus & Scae & Scae_2 \\
\hline Streptomyces coelicolor A3(2) & Scoe & Scoe_l \\
\hline Streptomyces lavendulae & Slav & \\
\hline Streptomyces pristinaespiralis & Spri & \\
\hline Streptomyces roseochromogenes subsp. Oscitans & Sros & Sros_l \\
\hline Streptomyces toyocaensis strain 7 & Stoy & \\
\hline Sulfolobus solfataricus P2 & Ssol & \\
\hline Sulfolobus tokodaii strain 7 & Stok & \\
\hline Synechococcus sp. WH8I02 & SYNE_I & SYNE_I \\
\hline Synechococcus sp. PCC7002 & SYNE_2 & \\
\hline Synechocystis sp. PCC6803 & SYNE_3 & SYNE_3 \\
\hline Thermobifida fusca & Tfus & \\
\hline Thermosynechococcus elongates BP-I & Telo & \\
\hline Trichodesmium erythraeum IMSIOI & Tery & \\
\hline Tropheryma whipplei TW08/27 & Twhi & \\
\hline Vibrio cholerae OI biovar eltor strain NI696I & Vcho & \\
\hline Vibrio parahaemolyticus RIMD 2210633 & Vpar & \\
\hline Wolinella succinogenes DSM I 740 & Wsu & \\
\hline Xanthomonas campestris pv. campestris strain ATCC 33913 & Xcam & \\
\hline Xylella fastidiosa $9 \mathrm{a} 5 \mathrm{c}$ & Xfas & \\
\hline Yersinia enterocolitica (type $0: 8$ ) & Yent & \\
\hline Zymomonas mobilis subsp. mobilis ZM4 & Zmob & \\
\hline
\end{tabular}

aThe system of acronym usage is: the first letter (capital) is the first letter of the genus followed by the first three letters (lower-case) of the species. If there is no species designation the first four letters of the genus are used (all in capitals). Redundant 4-letter acronyms are distinguished by unique following numbers. See [74] for a comprehensive listing with hyperlinks to the Taxonomy database records and the GenBank records at NCBI. 


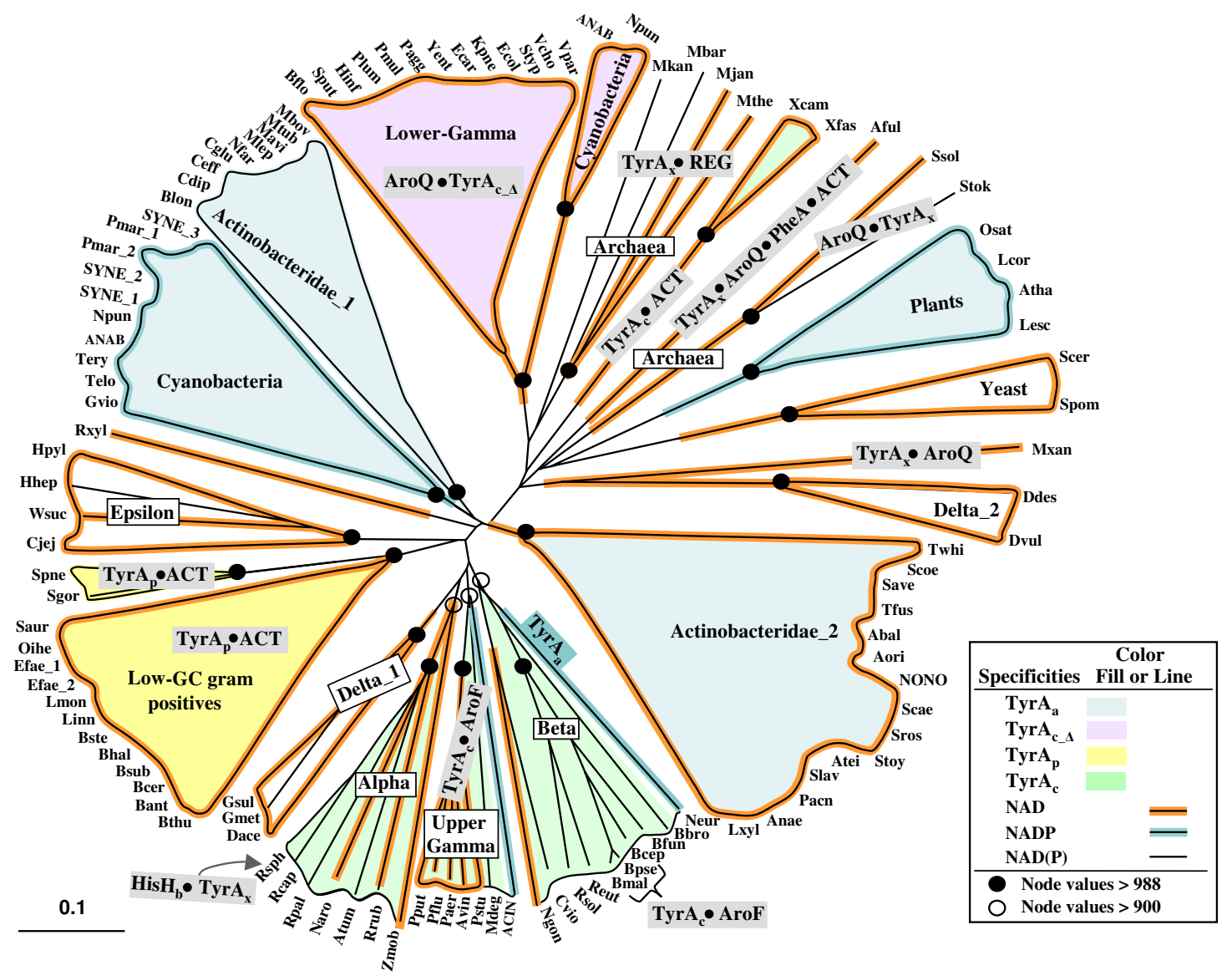

Figure 2

Phylogenetic tree for trimmed core domains of selected members of the TyrA Superfamily. Acronyms used for the various organisms are given in alphabetical order in Table 2. (A more extensive listing that includes organisms not shown in Fig. 2 and which also is hyperlinked to all of the individual GenBank records is given in Table SI. A similar table that also includes compilation of known and predicted substrate specificities is maintained at AroPath [73]. Lineages possessing experimentally established TyrA $A_{a}$, TyrA $A_{p}$ TyrA $_{c}$ or TyrA $A_{c} \Delta$ proteins are indicated by fill colors specified in the legend. Three specificity patterns for the pyridine nucleotide substrate are shown by line colors (see figure box). Although the cyanobacteria are depicted as having NADP $^{+}$-specific TyrA proteins, some of them can also accept NAD, albeit to a lesser degree. All proteins having an aspartate

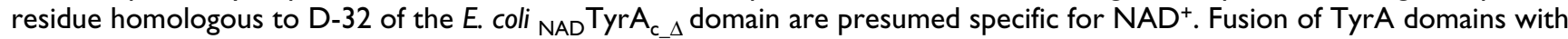
other catalytic domains is indicated within grey boxes (AroQ•TyrA, TyrA•AroF, HisH $\mathrm{H}_{\mathrm{b}} \cdot \mathrm{TyrA}$, and TyrA・AroQ・PheA・ACT) using the convention of a bullet to represent the interdomain area. The boxes overlap any relevant lineages. TyrA proteins having carboxy-terminal fusions with regulatory domains (TyrA•ACT and TyrA•REG) are also shown. The distance scale bar at the bottom left represents substitutions per site.

other segments of aromatic biosynthesis (unpublished data), we find it useful to separate "upper-gamma" Proteobacteria from "lower-gamma" Proteobacteria (an "enteric lineage" with Shewanella oneidensis as approximately the most divergent member). This separation is because the beta Proteobacteria and the upper-gamma
Proteobacteria exhibit a smooth continuity of relatively few evolutionary events with respect to aromatic biosynthesis, in striking contrast to extraordinarily dynamic evolutionary events in the lower-gamma Proteobacteria. As a consequence, the lower-gamma Proteobacteria are much more distinct (in terms of aromatic biosynthesis) from the 
upper-gamma Proteobacteria than the upper-gamma are from the beta Proteobacteria.

Figure 2 shows that alpha, beta and epsilon divisions of Proteobacteria form phylogenetically coherent clusters with respect to their TyrA proteins. Although delta Proteobacteria fall into two well-separated groupings denoted as Delta_1 and Delta_2, this should not be surprising since these groupings diverge at a deep level on the 16S rRNA tree where genome representation is poor. In addition, the Myxococcus xanthus TyrA sequence, currently an orphan (three o'clock in Fig. 2), represents a third divergent lineage in delta Proetobacteria. In contrast to delta Proteobacteria, genomic representation for the gamma Proteobacteria is relatively good. Nevertheless their TyrA sequences separate into several well-spaced groupings, albeit for entirely different reasons. In this case, the split seen between two clades of these fairly close relatives (upper-gamma and lower-gamma) is attributed to particularly dynamic evolutionary events compressed into a relatively short time span in the lower-gamma Proteobacteria. (We refer to such a dynamic divergence as an evolutionary jump; see the next section.) Note that the allocation of upper-gamma and lower-gamma Proteobacteria to separate TyrA congruency groups is not the same as being incongruent. It is quite possible that as new genomes come on line, new and intermediate TyrA sequences may result in the merging of the foregoing two congruency groups (currently tyrosine congruency group 1 (TyrCG-1) and tyrosine congruency group 2 (TyrCG-2)).

\section{Comparison of tryptophan and tyrosine congruency groups}

Although the true extent of lateral gene transfer (LGT) at present must be described as intensely controversial, there is little doubt that any given organism is mosaic with respect to some unknown fraction of its gene repertoire. Our "accounting" system for keeping track of proteins that are faithful to the vertical genealogy is to formulate congruency groupings that are defined by congruence of given protein-tree clusters to a section of the 16S rRNA tree. Ultimately this information will reveal which organisms are "pure" with respect to the vertical inheritance of a given pathway or pathway segment. Our congruency groups are intended to be fluid, in that with the continued availability of new sequences, a previous orphan sequence may very well become the seed for a new congruency group. On the other hand, previously separate congruency groups have the potential to merge. (See Methods for more information.) The present tyrosine congruency groups are listed on the AroPath website [9].

Seven tryptophan congruency groups in Bacteria were previously formulated [8] based upon the correspondence of cohesive clusters in trees of Trp-protein concatenates with sections of $16 \mathrm{~S}$ rRNA trees. The information input for for- mulation of tryptophan congruency groups is of greater quality than for tyrosine congruency groups because seven-protein concatenates could be used for the former. On the other hand, the broad information input supporting tyrosine congruency groups in this study is more comprehensive because of greater genome availability. Tryptophan congruency group 1 (TrpCG-1) corresponds perfectly with the organisms represented in TyrCG-1, these being the lower-gamma Proteobacteria (enteric lineage). The upper-gamma Proteobacteria (TyrCG-2) and the beta Proteobacteria (tyrosine congruency group 3; TyrCG-3) are represented by different tyrosine congruency groups. In contrast, the membership of tryptophan congruency group 2 (TrpCG-2) includes both the uppergamma Proteobacteria and the beta Proteobacteria. The latter merging probably reflects the advantage conferred by the greater information content of the concatenated sequences used to define tryptophan congruency groups.

Species of Xylella and Xanthomonas are usually referred to as gamma Proteobacteria. They probably represent an outlying deeply branching lineage, although trees based on concatenated strings of proteins [10] or 16S rRNA [11] position them with beta Proteobacteria. In any event, Trpprotein concatenate trees placed Xylella and Xanthomonas within TrpCG-2, which contains both upper-gamma and beta Proteobacteria. In contrast, the TyrA domains from Xylella and Xanthomonas were well separated (at about two o'clock in Fig. 2) from those of any other organism. This might simply be due to the limited resolving power of a single protein in combination with too few close relatives. (Note that single Trp-protein trees sometimes failed to achieve the congruency-group placements that were resolved by seven-protein Trp concatenates [8]). An additional clue may be relevant. The TyrA proteins from the Xylella/Xanthomonas genera possess an ACT domain, which has not been observed in any other proteobacterial TyrA proteins thus far. In view of this, origin by LGT seems to be a distinct possibility, but with the important caveat that no likely genome donors are yet obvious on the criterion of sequence similarity. Perhaps more likely is the following possible explanation that postulates a basis for accelerated divergence. The TyrA domains of Xanthomonas/Xylella proteins have an indel structuring (insertions and/or deletions) that places them within the TyrA $_{c \_\Delta}$ specificity subclass (see below). We suggest (see below) that such indel structuring reflects interaction of the core TyrA domain with an extra-domain extension. Thus, selection for amino acid changes accomplishing a new domain-domain interaction could account for accelerated divergence of the Xanthomonas/Xylella sequences on the TyrA tree (Fig. 2).

Cohesive tryptophan congruency groups of the alpha Proteobacteria (tryptophan congruency group 3; TrpCG-3) 
Table 3: Curated TyrA amino-acid sequence files at AroPath [35]

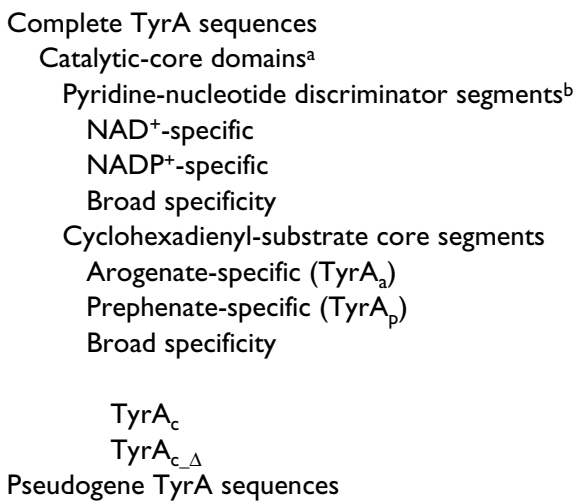

aTrimmed free of $\mathrm{N}$-terminal or $\mathrm{C}$-terminal extensions, including any fusions with regulatory domains or other catalytic domains.

bHigh-glycine $\beta \alpha \beta$ Rossmann fold at the $\mathrm{N}$-terminus.

and the cyanobacteria (tryptophan congruency group 4; TrpCG-4) match up well with the corresponding tyrosine congruency groups (tyrosine congruency group 4 (TyrCG4) and tyrosine congruency group 8 (TyrCG-8), respectively). The TyrA proteins of epsilon Proteobacteria define a cohesive tyrosine congruency group (tyrosine congruency group 5; TyrCG-5), whereas the Trp-protein concatenates of epsilon Proteobacteria did not exhibit a coherent congruency group, due at least in part to LGT [8]. The delta Proteobacteria separate into two distinct tyrosine congruency groups: Delta_1 (tyrosine congruency group 6; TyrCG-6) and Delta_2 (tyrosine congruency group 7; TyrCG-7), as shown in Fig. 2. It is likely that corresponding tryptophan congruency groups exist (work in progress), but at the time of the Xie et al. study [8] only Trp-pathway protein concatenates for Desulfovibrio vulgaris (Delta_2) and Geobacter sulfurreducens (Delta_1) were available, and they were provisionally listed as "orphans". In the present work TyrA sequences from Deinococcus radiodurans and Thermus thermophilus are the sole members of tyrosine congruency group 12 (TyrCG-12). At the time of the Trp-pathway work, the genome of Thermus was unavailable and the Deinococcus concatenate was listed as an orphan. It is expected that the Deinococcus and Thermus concatenates will now seed a new tryptophan congruency group.

Whereas tryptophan congruency group 5 (TrpCG-5) is defined by cohesive concatenates from actinomycete bacteria, the TyrA proteins from the same organisms separated into two distinct congruency groups. It is intriguing that this partitioning into two congruency groups correlates with narrowed specificity for $\mathrm{NAD}^{+}$(indicating an evolutionary jump) in one of the groups. The latter group (tyrosine congruency group 11; TyrCG-11) is denoted
Actinobacteridae_2 in Fig. 2, whereas tyrosine congruency group 10 (TyrCG-10) is displayed as Actinobacteridae_1. The opposite scenario whereby a single tyrosine congruency group corresponds to split tryptophan congruency groups applies in the case of low-GC gram-positive bacteria. Whereas TyrA proteins form a single congruency group in these organisms (tyrosine congruency group 9; TyrCG-9), a small cluster of Trp-pathway concatenates from Bacillus subtilis, B. stearothermophilus, and B. halodurans (tryptophan congruency group 6; TrpCG-6) separate distinctly from the remaining organisms (tryptophan congruency group 7; TrpCG-7). The latter evolutionary jump reflects a dynamic scenario of tryptophan-pathway evolutionary events that include loss of one gene from the trp operon, insertion of the trp operon into a 6-gene aro operon to produce a supraoperon, and acquisition of the TRAP (tryptophan-activated RNA-binding protein) mechanism of regulation by an RNA-binding protein [7].

Tyrosine congruency groups and tryptophan congruency groups are maintained and updated at the AroPath website [12].

\section{Distribution in nature of TyrA specificity subclasses for the cyclohexadienyl substrate}

Four qualitative classes of specificity for the cyclohexadienyl substrate populate the TyrA superfamily of homologs (Fig. 1). These include PPA-specific $\left(\operatorname{Tyr}_{\mathrm{p}}\right), \mathrm{AGN}$-specific $\left(\operatorname{TyrA}_{\mathrm{a}}\right)$, the broad-specificity cyclohexadienyl $\left(\operatorname{TyrA}_{\mathrm{c}}\right)$ dehydrogenases and a fourth class represented by an enzyme of antibiotic biosynthesis (PapC) that converts 4amino-4-deoxy-prephenate to 4-amino-phenylpyruvate [13]. Representatives of each specificity class have been studied at molecular and genetic levels. TyrA family members sharing a given substrate specificity do not necessarily cluster tightly together, and assignment of substrate specificity to experimentally uncharacterized TyrA homologs is uncertain unless they exhibit very high amino acid identities with experimentally characterized TyrA proteins. In some cases we do not accept older literature reports without more recent verification. For example, the yeast $S a c$ charomyces cerevisiae $\operatorname{Tyr}_{\mathrm{x}}$ was characterized as a $\operatorname{TyrA}_{\mathrm{p}}$ protein [14] long before it was recognized [15] that PPA preparations were often contaminated with AGN (an unknown compound at that time).

Our collection of curated TyrA sequences at AroPath (see Table 3) contains trimmed sequences that comprise catalytic-core domains. This collection was divided into two groups based on whether the sequences contained the relatively short $\mathrm{N}$-terminal pyridine-nucleotide discriminator segment or the longer C-terminal cyclohexadienylsubstrate core segment. The sequences in the latter group were assembled into subgroups representing established substrate specificities $\left(\operatorname{TyrA}_{a^{\prime}} \operatorname{TyrA}_{\mathrm{p}}\right.$ and $\left.\operatorname{Tyr} \mathrm{A}_{c}\right)$ and were 


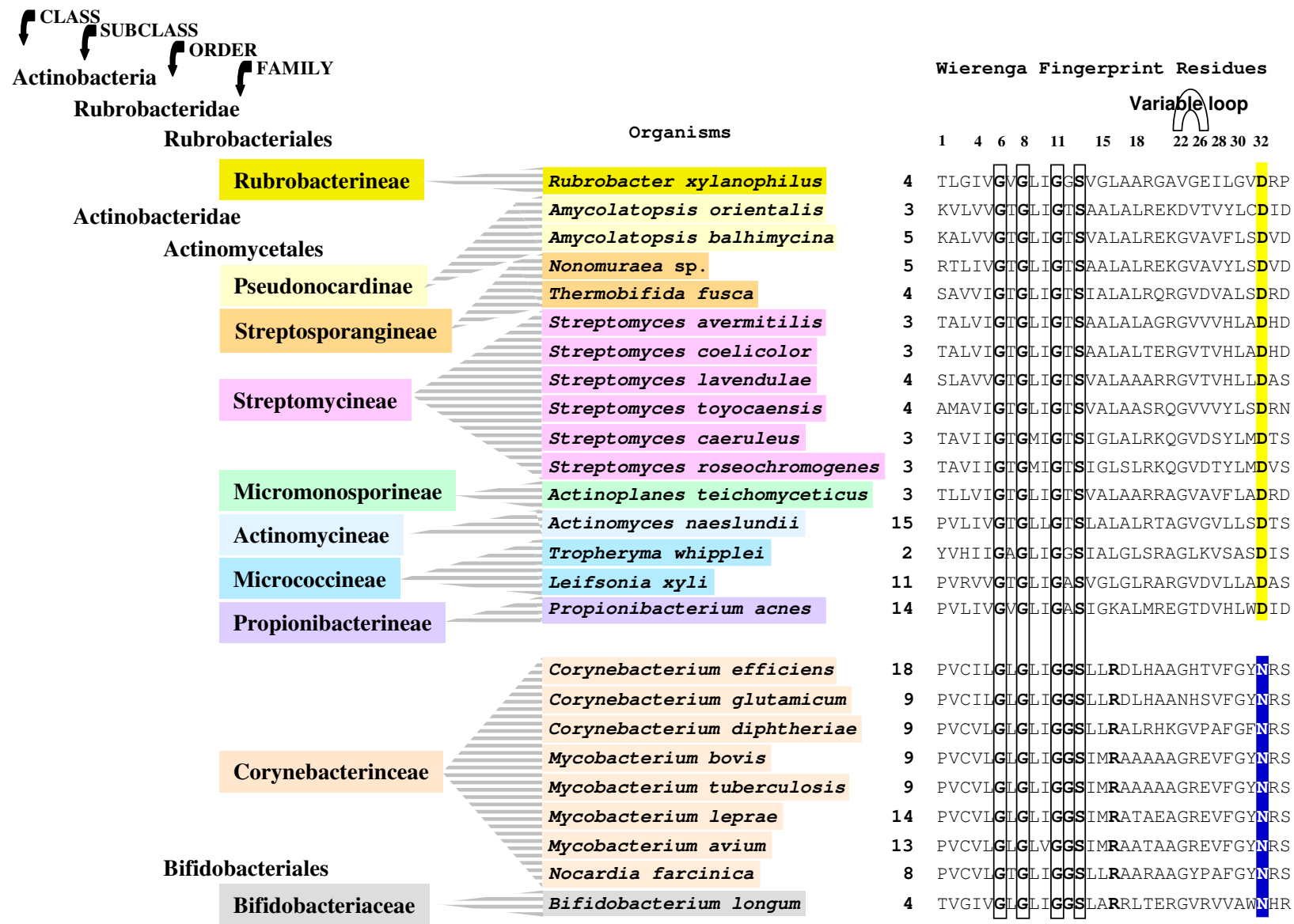

\section{Figure 4}

Alignment of the $\mathrm{N}$-terminal glycine-rich P-loop of TyrA•ACT proteins from the Class Actinobacteria. These are specific for $L-$ arogenate as substrate, but fall into two groups with respect to the pyridine nucleotide co-substrate. The top NAD ${ }^{+}$-specific group possesses an aspartate (D) at position 32 ( $E$. coli numbering), whereas the bottom $\mathrm{NAD}^{+} / \mathrm{NADP}^{+}$group possesses an asparagine at the homologous position. Residue numbers are shown at the left. The species in the middle are color coded to match the hierarchical taxon positions obtained from NCBI. The variable loop of the Wierenga fingerprint [26], which in E. coli contains five residues (22-26), contains the minimal two residues in all of the Actinobacteria shown. The organisms on the right are color coded according to the taxonomic position indicated on the left (NCBI). The Rubrobacter xylanophilus TyrA sequence is an orphan in the tree displayed in Fig. 2, as consistent with its outlying position in the taxonomy scheme.

query from N. europaea (March,2005) is the protein from Ralstonia solanacearum ( $48 \%$ identity), which is known to possess broad specificity for both of its substrates (i.e., $\operatorname{NAD}(\mathrm{P})$ TyrA $)[21,22]$.

The TyrA sequences of Actinobacteria separate into two distinct groupings on the protein tree (Fig. 2). Coryneform bacteria in one sub-cluster have been rigorously characterized as the $\mathrm{NAD}(\mathrm{P})_{\mathrm{TyrA}}$ substrate specificity type. On the other hand, a variety of Streptomyces species have been shown $[23,24]$ to possess ${ }_{\mathrm{NAD}} \mathrm{TyrA}_{\mathrm{a}}$, and TyrA proteins of these organisms populate the second Actinobacteria subcluster of Fig. 2. Figure 4 shows sequence alignments of the N-terminal pyridine-nucleotide discriminator regions of currently available actinomycetes. The conserved 'D' residue (highlighted in yellow) in the upper group is a reliable indicator of $\mathrm{NAD}^{+}$specificity, in part because $\mathrm{NADP}^{+}$is repelled by the negative charge at this position. 
The asparagine residue (highlighted in blue) in the corresponding position in members of the lower group indicates NAD $(\mathrm{P})^{+}$specificity as discussed by Bonner et al. [25]. Rubrobacter xylanophilus is the most distant representative of the Actinobacteria, being the sole member of the subclass taxon Rubrobacteridae, and its protein (denoted Rxyl) appears as an orphan in Fig. 2.

A similar relationship of phylogenetic separation associated with narrowed specificity for pyridine-nucleotide substrate exists for the low-GC gram-positive bacteria (eight o'clock in Fig. 2). Here the major clade is $\mathrm{NAD}^{+}$specific, whereas species of Streptococcus have retained the ancestral breadth of specificity for $\mathrm{NAD}^{+} / \mathrm{NADP}^{+}$. Alignments of the pyridine-nucleotide discriminator regions of these latter two groups match up extremely well with the upper alignment of Fig. 4 where residue 32 of the Wierenga fingerprint [26] is ' $\mathrm{D}$ ' and with the lower alignment where residue 32 is ' $\mathrm{N}$ ' (data not shown).

Recently, a plant tyr $A_{\mathrm{a}}$ from Arabidopsis thaliana has been reported to consist of two near-identical domains that are fused [27]. The gene encoding this $68-\mathrm{kDa}$ protein coexists in the genome with a single-domain paralog [28] that encodes a predicted $37-\mathrm{kDa}$ protein, somewhat larger than the catalytic-core domain of TyrA $\mathrm{a}_{\mathrm{a}}$ rom Synechocystis. TyrA $_{a}$ (known to be located in higher-plant chloroplasts [2]) may have originated from cyanobacteria via endosymbiosis. If so, however, the plant $\mathrm{TyrA}_{\mathrm{a}}$ sequences have diverged sufficiently that they no longer share a specific phylogenetic grouping with the cyanobacterial TyrA sequences. This is in marked contrast with the phylogenetic coherence of the tryptophan synthase subunit proteins (TrpEa and TrpEb_1) from cyanobacteria and higher plants [29].

\section{Prephenate dehydrogenases}

TyrA $\mathrm{p}_{\mathrm{p}}$ is conspicuously represented by a large clade of lowGC gram-positive organisms, of which Bacillus subtilis $\operatorname{TyrA}_{p}$ is the best studied [30]. Thus far, all TyrA proteins are fused to a C-terminal ACT domain, and therefore no "minimal" TyrA proteins that consist only of a catalytic core are available as yet. At the level of physiological function, it should be added that those cyclohexadienyl dehydrogenases that exhibit a very substantial preference for prephenate are for all practical purposes prephenate dehydrogenases, even though they carry a formal designation of TyrA $A_{c}$ or TyrA $A_{c}$. These include most, if not all, of the AroQ $\bullet$ TyrA $A_{c \Delta}$ enzymes of the enteric lineage (lowergamma in Fig. 2). The TyrA $\mathrm{A}_{c}$ protein from Neisseria gonorrhoeae (and by inference, the closely related N. meningitides) is also a well-studied example of overwhelming preference for prephenate [21].

\section{PapC dehydrogenases}

PapC participates in the formation of $p$-aminophenylalanine as a step in the synthesis of at least two antibiotics (see Fig. 1). It is so far represented by only a few sequences. The PapC specificity is strongly indicated by absence of the otherwise invariant residue H197 (E. coli numbering) that is associated with recognition of a 4hydroxy moiety in the cyclohexadienyl substrates of the aforementioned dehydrogenases. This moiety, of course, differs in being a 4-amino substituent in the substrate used by the PapC dehydrogenase (Fig. 1). See Bonner et al. [25] for a more detailed overview.

\section{The "redundant" trp/aro supraoperon of Nostocl Anabaena}

All cyanobacteria possess a highly conserved $t y r A_{a}$ gene, as well as a complete suite of tryptophan-pathway genes that are dispersed (unlinked) in the genome. The largegenome cyanobacterial lineage consisting of the Nostoc and Anabaena genera possess in addition a unique and seemingly redundant trp/aro supraoperon consisting of most of the aforementioned genes [31]. These include a second tyrA gene (curated as $\operatorname{tyr} A_{c_{-} \Delta}$ ), six trp-pathway genes (all except $\operatorname{trpC}$ ), and genes encoding the first two common-pathway steps of aromatic amino acid biosynthesis. All of these supraoperonic genes appear to be redundant in that they are represented by homologs (paralogs or xenologs) elsewhere in the Nostoc and Anabaena genomes at scattered loci. The closest BLAST hits for the Nostoc/Anabaena $\operatorname{TyrA}_{\mathrm{c} \Delta}$ proteins are not the co-existing TyrA $_{a}$ homologs present in their own genomes (and universally present in cyanobacteria). Rather the closest BLAST hits are to the $\operatorname{Tyr}_{c_{\_} \Delta}$ domains of the AroQ $\bullet \operatorname{TyrA}_{c_{-} \Delta}$ fusions in the enteric lineage. Since the enteric proteins are $\mathrm{NAD}^{+}$-specific and strongly prefer prephenate, it is likely that the "extra" cyanobacterial proteins are also ${ }_{\mathrm{NAD}} \mathrm{TyrA}_{\mathrm{c} \_\Delta}$ proteins. Indeed, this would be consistent with enzymological evidence provided in the literature for both Nostoc and Anabaena [20].

Concerning the evolutionary origin of the redundant block of linked genes found in the Nostoc and Anabaena genomes, at least two possibilities await further illumination. (i) These genes might have been acquired by a common ancestor of Nostoc and Anabaena via lateral gene transfer. This is consistent with the observation that biosynthetic-pathway operons are generally absent in the cyanobacteria, and all of the linked genes could have been recruited in a single event. However, at present no candidate donor genomes are known that possess this supraoperon combination of genes. If the TyrA $\mathrm{c}_{\triangle} \Delta$ proteins of Nostoc/Anabaena and the enteric lineage are possibly related by LGT, it is of interest that the N-terminal extension of $\mathrm{TyrA}_{\mathrm{c} \Delta}$ from Nostoc/Anabaena resembles a degraded AroQ domain of $\mathrm{AroQ} \bullet \mathrm{Tyr}_{\mathrm{c} \_\Delta}$ from enterics. In 
both cases the $\mathrm{N}$-terminal residues may compensate for indel deletions within the catalytic core region of $\operatorname{TyrA}_{c_{\_} \Delta}$. Subsequently, AroQ function may have evolved in one lineage (or have been lost in the other). This possibility of domain-domain interaction is consistent with the established interdependence of the AroQ $\bullet$ and $\bullet T_{y r A} A_{c_{\triangle} \Delta}$ domains from E. coli [32]. Alternatively, $\operatorname{tyr} A_{a}$ and $\operatorname{tyrA}_{c \_\Delta} \Delta$ (and the duplicated trp and aro genes present in the supraoperon) might be ancient paralogs within the cyanobacterial lineage. If so, at a time following divergence of heterocystous cyanobacteria from the unicellular cyanobacteria, the latter may have lost the clustered block of aromatic-pathway genes in a single event of reductive evolution. The supraoperonic genes might be related to a specialized function associated with "developmental" physiological processes that typify the filamentous, heterocyst-forming cyanobacteria. This might be reminiscent of the nature of the phenazine-pigment operon of Pseudomonas aeruginosa. Here unique phenazine-pathway genes are combined with a redundant gene of commonpathway aromatic biosynthesis and two redundant (and fused) genes of tryptophan biosynthesis. This accomplishes the linkage of specific phenazine biosynthesis with a supply of 2-amino-2-deoxy-isochorismate, the branchpoint of divergence toward phenazine and tryptophan $[33,34]$. This complexity in which multiple paralogs are differentially deployed is consistent with the large genome sizes of Anabaena (7.2 MB) and Nostoc (9.2 MB), compared with the much smaller unicellular genomes of Prochlorococcus marinus (1.7 MB), Synechococcus sp. WH8102 (2.4 MB), and Synechocystis sp. PCC6803 (3.6 $\mathrm{MB})$.

\section{Profile hidden Markov models (HMMs) to distinguish specificity subfamilies for cyclohexadienyl substrate}

The limited information thus far available about specific molecular roles of particular TyrA amino acid residues has been summarized recently [25]. The catalytic-core domains of known $\operatorname{TyrA}_{\mathrm{a}^{\prime}} \operatorname{TyrA}_{\mathrm{p}^{\prime}} \operatorname{TyrA}_{\mathrm{c}^{\prime}}$ and $\operatorname{TyrA}_{\mathrm{c} \Delta \Delta}$ proteins were selected from our files of TyrA catalytic-core domains [35], and a new subset of sequences was prepared that lacked the pyridine nucleotide discriminator segment, a glycine-rich $\beta \alpha \beta$ region at the $\mathrm{N}$ terminus. Although the glycine-rich $\beta \alpha \beta$ region is not the only segment that contacts pyridine nucleotide substrate, it is the sole region that discriminates between $\mathrm{NAD}^{+}$and $\mathrm{NADP}^{+}$. The resulting trimmed sequence is defined as the "cyclohexadienyl-substrate core segment". No distinctive motifs were found that, in isolation, would be a clear predictive indicator of specificity for cyclohexadienyl substrate. Similar substrate specificity profiles probably can be dictated by alternative patterns of interplay between different residue combinations.
Because of the rapid accumulation of incorrectly annotated TyrA entries in GenBank and other databases, partly due to the complications of misnaming that are associated with gene fusions and partly to a failure to assimilate published substrate specificities, the use of BLAST does not return reliable annotations with respect to substrate specificity. Even the HMMs used in Pfam [36] and Interpro [37] were not helpful in this case because the HMM deployed in those databases was broadly but incorrectly defined as 'prephenate dehydrogenase ( $\left.\mathrm{NADP}^{+}\right)$activity' for all TyrA dehydrogenases (accession number PF02153 in Pfam and entry IPR003099 in Interpro). However, Profile HMM is known to be well suited for modeling a particular sequence family of interest and for finding additional remote homologs [38]. It is reputed to outperform methods that rely only upon pair-wise alignment of homologous residues in predicting protein function [39]. Therefore, profile HMMs were constructed using our multiple sequence alignments of each curated TyrA specificity subfamily, using the HMMER package [38].

The profile HMMs obtained are only tentatively reliable for prediction of substrate specificity. To facilitate ongoing and future functional annotations, we have made our profile HMMs available as a working resource for "specificity prediction" at AroPath [40]. Users can match query sequences against the four profile HMMs to predict the subfamily to which a query sequence belongs. It is anticipated that future experimental data relevant to substrate specificity will facilitate refinement of the prediction program. For example, at present the program predicts that the TyrA sequences from organisms such as Helicobacter pylori and Saccharomyces cerevisiae belong to the Tyr $\mathrm{A}_{\mathrm{a}}$ grouping, and it will be interesting to see whether this holds up to experimental confirmation. It is additionally fascinating that (i) the dehydrogenase from Archaeoglobus fulgidus is predicted to belong to the indel-containing $\mathrm{TyrA}_{\mathrm{c} \_\Delta}$ grouping and (ii) that it possesses a possible cooperatively interacting extra-core domain extension (an AroQ fusion), just as occurs for the large clade of enteric bacteria. If this is relevant, it is even more fascinating that the Archaeoglobus aroQ is fused at the C-terminal side of $\operatorname{tyr}_{c_{\perp} \Delta}$, rather than at the $\mathrm{N}$ terminus as is the case with enteric bacteria.

Users at AroPath [41] can enter query sequences into interactive multiple sequence alignments with any of the four sets of "cyclohexadienyl-substrate core segments" sequences that were used to train the profile HMMs. An ongoing effort is in process to extend the predictor capability to include the pyridine nucleotide substrate as well. One can also align query sequences of interest with either an assemblage of the complete set of curator-approved TyrA catalytic-core TyrA sequences or with any desired subset of seed sequences. 
Table 4: Cyclohexadienyl substrates and inhibitors of TyrA proteins possess identical sidechains

\begin{tabular}{|c|c|c|c|c|}
\hline Organism & Co-substrate & Substrate(s) & Inhibitor(s) ${ }^{\mathrm{a}}$ & Reference \\
\hline Synechocystis sp. & $\mathrm{NADP}^{+}$ & AGN & TYR & [25] \\
\hline Arabidopsis thaliana & $\mathrm{NADP}^{+}$ & AGN & TYR & {$[27,28]$} \\
\hline Nitrosomonas europaea & $\mathrm{NADP}^{+}$ & AGN & None & {$[2 I]$} \\
\hline Corynebacterium glutamicum & $\mathrm{NAD}(\mathrm{P})^{+}$ & AGN & None & {$[42,43]$} \\
\hline Neisseria gonorrhoeae & $\mathrm{NAD}^{+}$ & PPA $^{b}$ & HPP & {$[21]$} \\
\hline Pseudomonas stutzeri & $\mathrm{NAD}^{+}$ & PPA/AGN & HPP/TYR & [I] \\
\hline Pseudomonas aeruginosa & $\mathrm{NAD}^{+}$ & PPA/AGN & HPP/TYR & [17] \\
\hline Zymomonas mobilis & $\mathrm{NAD}^{+}$ & PPA/AGN & None & [18] \\
\hline
\end{tabular}

aAbbreviation: HPP, 4-hydroxyphenylpyruvate. ${ }^{\text {'This }}$ TyrA $_{c}$ enzyme has an overwhelming preference for PPA, but will use AGN poorly.

\section{The catalytic-core domain of TyrA proteins}

The simplest set of fully functional TyrA proteins consists only of the catalytic-core domain (about 180 amino acids) [1] and includes the well-characterized $\operatorname{TyrA}_{c}$ enzymes from Neisseria gonorrhoeae [21] and Zymomonas mobilis [18], as well as TyrA from a cyanobacterium [25]. In addition the catalytic-core domain from Pseudomonas stutzeri has been engineered for study from a $\operatorname{tyr}_{c} \bullet$ aroF fusion [1]. These model core proteins are roughly as divergent from one another on the TyrA protein tree as are the organisms that contain them (Fig. 2). In view of the possibility raised in this paper about inter-domain interactions, the single-domain TyrA proteins are undoubtedly the simplest sources for study of the fundamental properties of the catalytic-core domain.

Xie et al. [1] suggested that in the set of catalytic-core TyrA proteins, inhibitors bind at the catalytic site and exhibit classical competitive inhibition with respect to the particular cyclohexadienyl substrates that can be accepted by a given organism. This model predicts that the specificity for the sidechains of substrates used would parallel the specificity for inhibitor sidechains. The information summarized in Table 4 supports this expectation. Thus, the TyrA $_{c}$ proteins of $P$. stutzeri and $P$. aeruginosa will accept either a pyruvyl (as with PPA) or an alanyl (as with AGN) sidechain in the alternative substrates used, and this is paralleled by recognition of either a pyruvyl (4-hydroxyphenylpyruvate) or an alanyl (TYR) sidechain in the competent inhibitor structures. In another case, the $N$. gonorrhoeae $\mathrm{TyrA}_{\mathrm{c}}$ exhibits an overwhelming substrate preference for PPA, and consistent with the foregoing, is subject to inhibition by 4-hydroxyphenylpyruvate but not by TYR. A variety of analog inhibitor structures were used by Xie et al. [1] to show that the minimal structure for binding at the substrate-binding site of $P$. stutzeri $\operatorname{TyrA}_{c}$ is a six-membered ring with a 4-hydroxy substituent.

In contrast to the $\operatorname{TyrA}_{\mathrm{c}}$ proteins just described, the $Z$. mobilis $\operatorname{TyrA}_{c}$ is totally insensitive to inhibition by either 4- hydroxyphenylpyruvate or TYR. Since both of these compounds lack a 1-carboxy moiety, it is reasonable to assume that the 1-carboxy substituent present in the two substrates accepted may be required for binding at the catalytic center. Thus, although TyrA from $Z$. mobilis will accept the same two substrates as does the TyrA ${ }_{c}$ from $P$. stutzeri, the greatly different inhibition results suggest that $Z$. mobilis obeys more stringent rules for binding at the catalytic site (i.e., a ring carboxylate must be present).

Synechocystis sp. and Arabidopsis thaliana $\operatorname{TyrA}_{\mathrm{a}}$ proteins accept as a substrate only AGN, which has an alanyl sidechain. The ring-carboxylate moiety is evidently not absolutely required for binding since these $\operatorname{Tyr}_{\mathrm{a}}$ proteins can recognize TYR (alanyl sidechain) as a competitive inhibitor. In contrast, since $N$. europaea $\mathrm{TyrA}_{\mathrm{a}}$ is not inhibited by TYR, it resembles the Z. mobilis $\operatorname{Tyr}_{\mathrm{c}}$ in the putative requirement for a 1-carboxy substituent to secure successful binding at the catalytic site.

In summary, some TyrA proteins probably exercise greater discrimination in their requirement for a 1-carboxy moiety for binding at the catalytic site, and these are insensitive to competitive inhibition by the aromatic reaction products (which lack the 1-carboxy substituent). Other TyrA proteins that require the 1-carboxy moiety for the fundamental catalytic process, but presumably do not require it for binding, will recognize product inhibitors that have the same sidechain as any substrate recognized.

\section{Specificity for the pyridine nucleotide co-substrate within the TyrA superfamily}

$\mathrm{NAD}^{+}$differs from NADP+ ${ }^{+}$only in that $\mathrm{NADP}^{+}$has a phosphate group esterified at the 2'-position of adenosine ribose. Therefore, the ability of a dehydrogenase to discriminate between those two lies in the particular enzyme region that contacts the ribose moiety. The glycine-rich region known to constitute the ADP-binding $\beta \alpha \beta$ fold is well known to be this point of contact [26]. This Rossmann $\beta \alpha \beta$ fold is inevitably positioned at the extreme $\mathrm{N}$ 
terminus of TyrA proteins, and the typical GXGXXG motif is almost always observed, as illustrated in Fig. 4. This region is helpful for assessment of probable specificities for pyridine nucleotide. One can be fairly sure that TyrA proteins possessing D-32 (E. coli numbering, reference [26]) are $\mathrm{NAD}^{+-}$-specific. A negatively charged residue (D or E) at position 32 is critical for hydrogen binding to the diol group of the ribose near the adenine moiety in $\mathrm{NAD}^{+}$ specific enzymes. $\mathrm{NADP}^{+}$-specific dehydrogenases cannot tolerate a negatively charged residue at position 32. TyrA proteins that possess an asparagine residue in the corresponding position appear to be broadly specific for both $\mathrm{NAD}^{+}$and NADP ${ }^{+}$as discussed above. No clearcut motif has been identified for $\mathrm{NADP}^{+}$-specific TyrA proteins, although at least one positively charged residue is expected in the region just beyond residue 32. By elimination, those sequences lacking D-32 or N-32 are strong candidates for $\mathrm{NADP}^{+}$specificity. As with the cyclohexadienyl co-substrate, narrowed specificity for $\mathrm{NAD}^{+}$(or $\mathrm{NADP}^{+}$) also seems to have occurred independently on many occasions (some examples given earlier).

The absolute specificity of TyrA $\mathrm{p}_{\mathrm{p}}$ proteins for PPA tends to be accompanied by absolute specificity for $\mathrm{NAD}^{+}$, as illustrated by the large Bacillus/Staphylococcus/Listeria/Enterococcus clade at eight o'clock in Fig. 2. However, it is interesting that species of Streptococcus have retained the presumed ancestral breadth of specificity for the pyridine nucleotide substrate. The opposite relationship, whereby absolute specificity for AGN tends to be accompanied by absolute specificity for $\mathrm{NADP}^{+}$, is also observed. Here three of the four $\operatorname{TyrA}_{\mathrm{a}}$ lineages described earlier exhibit this pattern. Exceptions, though, are the aforementioned $\mathrm{TyrA}_{\mathrm{a}}$ proteins of Actinobacteridae_1 which accept either $\mathrm{NAD}^{+}$or NADP+, as well as the TyrA ${ }_{\mathrm{a}}$ proteins of the sister Actinobacteridae_2 which are specialized for $\operatorname{NAD}^{+}[42,43]$.

The $\operatorname{TyrA}_{c}$ proteins of most complete-genome organisms thus far have happened to be $\mathrm{NAD}^{+}$-specific, and this has been the property of the most rigorously characterized ones (from Z. mobilis, P. stutzeri, and P. aeruginosa). However, it is clear from extensive enzymological surveys [22] that $\mathrm{TyrA}_{\mathrm{c}}$ proteins having broad specificity for $\mathrm{NAD}^{+}$ $\mathrm{NADP}^{+}$are common, examples including species of Ralstonia and Burkholderia. The spectrum of variation that can exist, even within a clade of organisms that are of fairly close relationship, is illustrated by one striking example. In the pseudomonad clade marked by a common $\operatorname{tyr} A \bullet \operatorname{aroF}$ fusion, the Acinetobacter sp. TyrA $\mathrm{c}_{\mathrm{c}}$ is $\mathrm{NADP}^{+}$-specific [44], whereas the sister subclade Pseudomonas/Azotobacter exhibits $\mathrm{NAD}^{+}$specificity (Fig. 2). Here the entire clade marked by a common ancestral fusion shares approximately the same profile of cyclohexadienyl substrate preference, but cofactor specificity has been narrowed in opposite directions.
We had previously suggested that there might be a general structural relationship of substrate pairing that tends to favor interaction between PPA and $\mathrm{NAD}^{+}$, on the one hand, and, on the other hand, between the greater positive charge of AGN and the greater negative charge of $\mathrm{NADP}^{+}$. These relationships may indeed be favored, but it increasingly appears that any combination can occur.

\section{Beyond the catalytic core: allosteric domains}

Various lineages have acquired an amino acid binding domain known as the ACT domain (pfam01842), which is known to bind a variety of amino acids, thus functioning as an allosteric domain for many proteins including phosphoglycerate dehydrogenase, aspartokinase, acetolactate synthase, phenylalanine hydroxylase, prephenate dehydratase and formyltetrahydrofolate deformylase. Recruitment of this domain by fusion with tyr $A_{p}$ appears to have occurred in a common ancestor of the large Bacillus/Staphylococcus/Listeria/Enterococcus/Streptococcus assemblage (Fig. 2). It is interesting that B. subtilis also possesses a gene encoding a free-standing ACT domain in its genome (incorrectly annotated as pheB). An additional fusion of genes encoding an ACT domain and tyrA (that arose independently, judging from the widely spaced tree positions) occurred in the common ancestor of Xanthomonas and Xylella. Actinobacteria usually possess a C-terminal extension that probably functions as an allosteric domain. The extension possessed by the Actinobacteridae_2 assemblage, which includes Streptomyces coelicolor and its relatives, appears to be an ACT domain. On the other hand, it is not all all clear that the C-terminal extension of the Actinobacteridae_2 assemblage is an ACT domain. This difference, in addition to the differing specificities for pyridine nucleotide substrate, may have contributed to the overall $\mathrm{TyrA}_{\mathrm{a}}$ divergence observed between the two Actinobacteridae groups. There is no correlation between presence of the ACT domain and specificity for cyclohexadienyl substrate since TyrA $\mathrm{A}_{\mathrm{p}}$ from the Bacillus clade is PPA-specific, Xanthomonas/Xylella TyrA is broadly specific, and Streptomyces $\mathrm{TyrA}_{\mathrm{a}}$ is AGN-specific.

B. subtilis, which belongs to the large clade having an ACT domain as a carboxy extension, has been extensively characterized [30]. 4-Hydroxyphenylpyruvate is an effective competitive inhibitor, as would be consistent with our proposed effects at the catalytic core for a PPA-specific enzyme. However, TYR, phenylalanine (PHE) and tryptophan were also inhibitors. The violation of the rule that the latter three amino acid inhibitors would not be expected to bind the catalytic core region (because they have alanyl sidechains even though the substrate-binding site only recognizes the pyruvyl sidechain of prephenate) and the finding that some of these were not competitive inhibitors can now be accounted for by the presence of the allosteric ACT domain. A carboxy extension shared by a 
number of Archaea (denoted 'REG' in Fig. 2) is presumably a regulatory domain as well. This is consistent with the recent result of Porat et al. [45] that not only 4-hydroxyphenylpyruvate, but also TYR, inhibited prephenate dehydrogenase activity of Methanococcus maripaludis.

\section{The tyrA gene is a popular fusion partner Fusion with aroQ}

tyrA may be fused with a number of other catalytic domains, each of them relevant to aromatic biosynthesis (Fig. 2). aroQ (encoding chorismate mutase) is frequently fused with a number of other aromatic-pathway genes [46]. The lower-gamma Proteobacteria (enteric lineage) located at twelve o'clock in Fig. 2 possess an aroQ•tyr $A_{c_{-} \Delta}$ fusion. The fusion physically links chorismate mutase (which forms PPA) with TyrA ${ }_{c_{\triangle} \Delta}$ (which utilizes PPA). The two protein domains of AroQ $\bullet T_{y r} A_{c \_\Delta}$ may have coevolved to produce cooperative protein-protein interactions since physical separation of the domains evoked relatively low activities of both activities in E. coli [32]. Substantial comparative work shows that the aroQ $\bullet t y r A_{c \_\Delta}$ fusion has been stably maintained throughout the entire enteric lineage [47]. Exceptions in some genomes lacking this fusion altogether can be attributed to reductive evolutionary loss in pathogens (e.g., Haemophilus ducreyi) or endosymbionts (e.g., Buchnera aphidicola). An independent $\operatorname{aro} \bullet \bullet t y r A$ fusion was generated in the common ancestor of Sulfolobus solfataricus and S. tokodaii (Fig. 2 ). Since the TyrA domain of Sulfolobus species lacks the indel structure of the TyrA $\mathrm{c}_{{ }_{\Delta} \Delta}$ class, it would be interesting to see whether physical separation of the two domains would yield evidence of independent function, in contrast to the results mentioned just above for E. coli.

\section{Fusion with aroF}

Secondly, $t y r A_{c}$ has been fused with aroF on at least two separate occasions in Bacteria. (The aroF gene encodes enolpyruvylshikimate-3-P synthase, the sixth enzyme in the common pathway of aromatic biosynthesis; see [5,6] for nomenclature used.) One clade includes members of the upper-gamma Proteobacteria: $P$. aeruginosa, $P$. syringae, P. putida, P. stutzeri, P. fluorescens and Azotobacter vinelandii. It is interesting that $P$. syringae has experienced a deletion of about 200 residues at the $\mathrm{N}$-terminal region of the AroF domain. This has been coupled with the acquisition of a stand-alone aroF gene that is absent in other members of the clade. Interestingly, the latter AroF shows high identity only with AroF from Agrobacterium tumefaciens, an alpha proteobacterium. The A. tumefaciens aroF, in turn, is unique compared to its $\alpha$-subdivision relatives, both in having divergent sequence and in being unlinked to $c m k$ and $r p s A$. Thus, it seems likely that the incongruence of AroF belonging to both P. syringae and $A$. tumefaciens reflects acquisition via LGT from some as yet unknown source. The disruption of the fused aroF domain in $P$. syringae is an unusual instance where the catalytic function of one fusion domain has become discarded while the function of the second domain has been retained. It is interesting to consider the possibility that the truncated remnant of the aroF fusion domain might be exploitable for use as an innovative source of a new regulatory domain. An additional fusion of tyrA with aroF has occurred independently within the beta Proteobacteria in the common ancestor of Burkholderia pseudomallei and B. mallei. This has been very recent since the closely related $B$. fungorum and B. cepacia organisms lack the fusion.

It has been suggested that presence of a given fusion may be useful for sorting out clades that diverged from a common ancestor, independent of other methods [48]. Different fusions offer the power of discriminating clades at various hierarchical levels, i.e., nested clades discriminated by nested gene fusions. The $\operatorname{tyr} \bullet \bullet \operatorname{aroF}$ fusion occurred in the common ancestor of the clade that includes the upper-gamma Proteobacteria shown in Fig. 2. One can reasonably assume that relatively close uppergamma organisms lacking the $\operatorname{ty} \mathrm{r} \bullet$ aroF fusion diverged from the common ancestor of the fusion clade prior to the fusion event. Such would appear to be the case, for example, with Acidithiobacillus ferrooxidans, an outlying member of the upper-gamma Proteobacteria that lacks the fusion. It is reasonable to conclude that the fusion event must have pre-dated the differential specialization for the pyridine nucleotide cosubstrate that distinguishes Acinetobacter sp. (NADP+-specific) from the large grouping of pseudomonads that are $\mathrm{NAD}^{+}$-specific.

\section{Fusion with his $\mathrm{H}_{b}$}

Thirdly, a single organism, Rhodobacter sphaeroides, possesses a $h i s H_{\mathrm{b}} \bullet$ tyrA fusion that must have occurred very recently. $h i s H_{\mathrm{b}}$ encodes an aromatic aminotransferase that is closely related to (or sometimes even synonymous with) imidazole acetol phosphate aminotransferase [49]. The his $H_{b} /$ tyrA/aroF linkage group is part of a supraoperon in some gram-negative bacteria in which a relatively conserved, yet frequently shuffled gene order is observed $[5,6]$. Hence, it is reasonable to assume that at the time just prior to fusion, his $H_{\mathrm{b}}$, tyrA and aroF were adjacent. Note that among the fusions currently known, his $H_{\mathrm{b}}$ and aroF are fused to the $\mathrm{N}$-terminal and C-terminal ends of tyrA, respectively. It would be interesting to know the substrate specificity of the $R$. sphaeroides TyrA domain. If it is AGN-specific the significance of $h i s H_{\mathrm{b}}$ presumably would be to transaminate PPA to form AGN, the substrate used by TyrA (see Fig. 1). On the other hand, if the dehydrogenase is PPA-specific, the significance of the $\mathrm{HisH}_{b}$ domain would be to transaminate the product of the $\mathrm{TyrA}_{\mathrm{p}}$ reaction. If the enzyme is a $\operatorname{TyrA}_{c}$ enzyme (as is probable), then $\mathrm{HisH}_{\mathrm{b}}$ likely is competent to catalyze either of the foregoing reactions. 


\section{Fusion with $A C T$}

The widespread ACT regulatory domain appears to have been acquired by independent fusions at least three separate times judging from the widely separated lineages that possess a TyrA•ACT fusion (Fig. 2). Xie et al. [5] initially noted homologous domains positioned at the $\mathrm{N}$ terminus of mammalian phenylalanine hydroxylase and at the $\mathrm{C}$ terminus of most microbial prephenate dehydratases. This domain is responsible for phenylalanine-mediated activation and phenylalanine-mediated inhibition of the hydroxylase and dehydratase enzymes, respectively. This domain was later named the ACT domain [50] and shown to be a widely distributed domain family that shares a conserved overall fold. Members of the ACT-domain family possess a wide variety of different ligand-binding capabilities. For example, the ACT domain of 3phosphoglycerate dehydrogenase binds $L$-serine as a allosteric inhibitor.

\section{Fusion with REG}

Another putative regulatory domain fused to tyrA (denoted $t y r A \bullet R E G$ ) is thus far restricted to some of the Archaea. This domain is a predicted regulatory domain, as described in COG4937.

\section{A novel 4-domain fusion}

Archaeoglobus fulgidus exhibits a striking four-domain fusion consisting of three catalytic domains and a regulatory ACT domain (TyrA $\bullet$ AroQ $\bullet P h e A \bullet A C T)$. The TyrA domain is predicted to belong to the $\operatorname{TyrA}_{c_{-} \Delta}$ class when used as a query input into the AroPath Specificity Predictor Tool [40]. We speculated earlier that the $\bullet$ AroQ fusion domain of Archaeoglobus may exercise cooperative interactions with $\operatorname{TyrA}_{\mathrm{c} \Delta}$, as appears to occur between the AroQ $\bullet$ TyrA $A_{c_{\_} \Delta}$ domains of $E$. coli and its relatives.

\section{tyrA in its syntenic context}

Although the genes of prokaryotes have clearly been subject to frequent scrambling, some gene-gene associations persist more tenaciously than others. Xie et al. $[5,6]$ asserted that one such ancestral gene string that has resisted scrambling forces is his $H_{b}>t y r A>\operatorname{aroF}$. As suggested above, contemporary gene fusions can serve as frozen-in-time indicators of ancient gene organizations that were later obscured by gene-scrambling events. Another gene string that is often within the syntenic region of $h i s H_{b}, t y r A$, and aroF is $c m k>r p s A$. Gene synteny in prokaryotes has not been easily recognized because substantial manual scrutiny in combination with a sufficient density of genomic representation on a given portion of the phylogenetic tree is necessary to detect patterns of synteny that are camouflaged by frequent scrambling events (inversion, deletion and transposition).
The domain Bacteria is now represented by a collection of sequenced genomes that is progressively approaching the genomic densities needed for meaningful analysis. Figure 5 provides a visual sense of the frequency with which $t y r A$ is closely positioned with other genes of aromatic biosynthesis, as well as the underlying patterns of overall synteny. These patterns are unstable, and yet persistent traces of synteny can be seen where genomic representation is sufficiently dense. The four genes of particular emphasis in this paper are color coded. Other genes that are engaged in aromatic biosynthesis are colored grey, and any other genes are white. At a very deep level of phylogenetic branching, Thermotoga exhibits a tyrA gene flanked by seven genes encoding all of the common steps of aromatic biosynthesis (two of them being fused). Since closely related genomes are not yet available here, we cannot judge whether these genes came together recently or whether an ancient pattern of synteny has been retained. Although tyrA is not linked to any functionally relevant genes in Aquifex, representing another point of deep phylogenetic branching, this does not necessarily mean that tyrA was not already generally associated with other aromatic-pathway genes at an early time. For reasons that are totally mysterious, certain scattered lineages exhibit a total lack of operon organization for aromatic-pathway genes (and indeed for most other biosynthetic pathways, such as that for histidine biosynthesis). These lineages (Fig. 5) include, besides Aquifex, those of Deinococcus, the actinomycetes, the cyanobacteria, and Chlorobium. Except for the actinomycetes, this phenomenon of total gene dispersal also applies to genes of tryptophan biosynthesis $[7,8]$.

When the various examples of $h i s H_{\mathrm{b}}>$ tyrA $>$ aroF linkage are mapped on a $16 \mathrm{~S}$ rRNA tree, they first appear in grampositive bacteria. In Bacillus and related organisms (such as Listeria), the his $H_{b}>t y r A>$ aroF unit is associated with a large ancestral operon consisting of $\operatorname{aroG}>\operatorname{aroB}>\operatorname{aroH}>$ his $\mathrm{H}_{b}>$ tyr $\mathrm{A}_{p}>$ aroF. Bacillus additionally possesses the $\mathrm{cmk}$ $>$ rps $A$ unit, albeit in a separate location. Interestingly, in one narrow subclade (B. subtilis, B. halodurans and $B$. stearothermophilus) the $\operatorname{trp}$ operon has been inserted between $\operatorname{aroH}$ and $h i s H_{b}$ to yield a supraoperon that has been fully characterized as a complex functional unit [51]. See Xie et al. [7] for a full presentation of evolutionary interpretation relevant to the latter. Though highly scrambled, a pattern of association of pheA with his $H_{\mathrm{b}}>\operatorname{tyr} \mathrm{A}$ $>a r o F$ is suggested by linkage patterns seen at the hierarchical level of Cytophaga and Bacteroides (Fig. 5). aroQ became associated with pheA through gene fusion as early as the divergence of the Spirochaetes to yield an aroQ $Q$ pheA $>$ tyrA $>$ aroF $>c m k>r p s A$ linkage unit (Leptospira interrogans in Fig. 5). The aroQ•pheA gene associated with tyrA and aroF in Clostridium difficile appears to have arisen from a distinctly different fusion event than that present in delta, epsilon, beta and upper-gamma Proteobacteria 


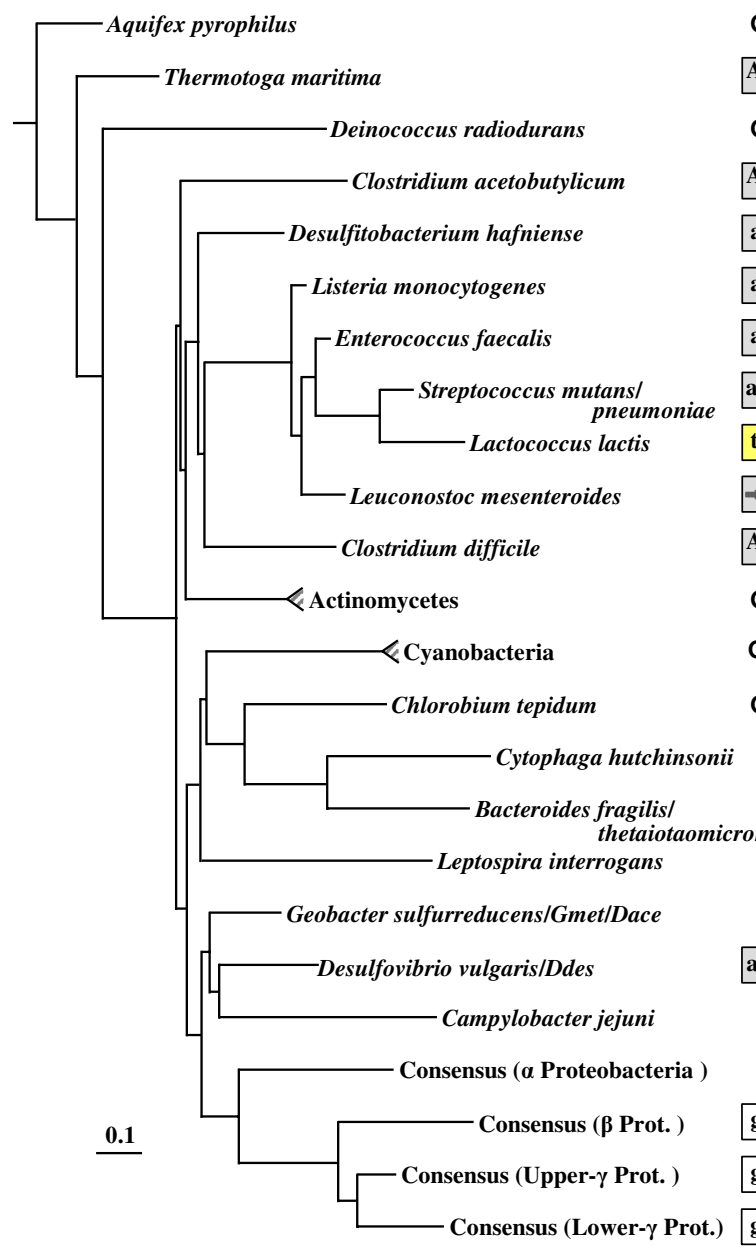

GENE DISPERSAL

$A_{\text {AroA }}$ tyrA aroF aroD aroG aroE・aroB $\operatorname{aroC}$ II

GENE DISPERSAL

AroA $A_{I B}>$ tyrA $>$ aroB $>$ aroF $>$ aroG aroQ*aroD aroE $>$ aroC

aroH AroA $_{\text {IB }}>$ tyrA $>$ aroF

aroG aroB aroH $>$ hisH $>$ tyrA $>$ aroF

aroD $\left.\quad \mathrm{ArOA}_{\mathrm{IB}}\right\rangle$ aroB aroG tyrA aroF aroE $>$ pheA

aroC $>$ aroD aroB aroG tyrA ORF aroF aroE pheA

tyrA aroF aroE pheA

$\Leftrightarrow \rightarrow \operatorname{Trp}$ Operon $\Rightarrow \rightarrow$ tkt $\Rightarrow$ aroD $A$ AroA $\rightarrow$ aroB aroF tyrA $>$ aroE $\operatorname{aroC_{1}}$

AroA $A_{I B}>\operatorname{aroB}>$ aroF $>$ aroG $>$ aroQ•pheA $>$ aroD $>$ aroE $>$ tyrA $>$

GENE DISPERSAL

GENE DISPERSAL

GENE DISPERSAL

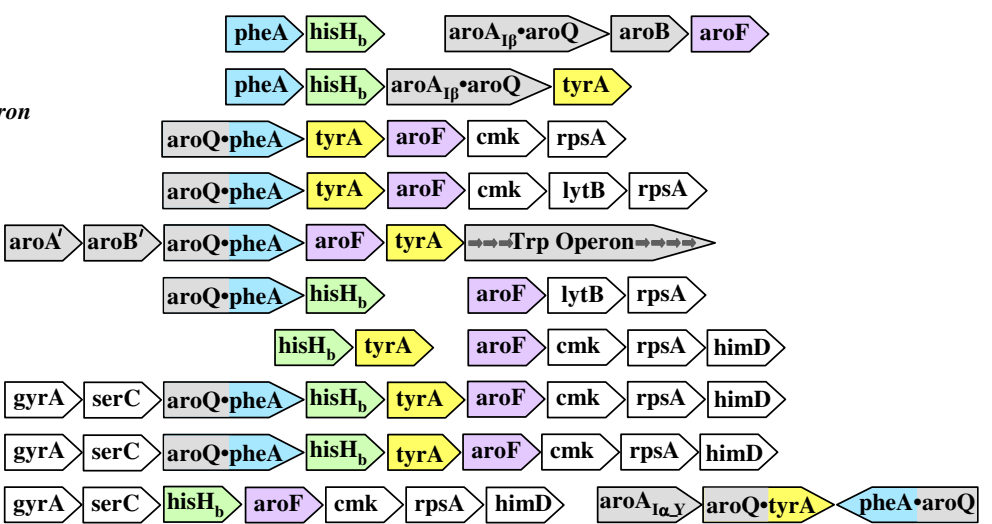

\section{Figure 5}

Context of gene organization for tyrA, profiled against the I6S rRNA tree of the domain Bacteria. pheA, his $\mathrm{H}_{\mathrm{b}}$, tyrA, and aroF are color coded. Lineages typified by complete dispersal of aromatic-pathway genes are indicated by "GENE DISPERSAL". Gmet refers to Geobacter metallireducens; Dace refers to Desulfuromonas acetoxidans; and Ddes refers to Desulfovibrio desulfuricans. Consensus gene organizations are shown for the alpha and beta divisions of the Proteobacteria. The gamma division is subdivided to yield consensus gene organizations for the upper- and lower-gamma (enteric lineage) organisms. Genes that are adjacent and share a common transcriptional direction appear to reside in operons (or supraoperons). Any white spacing indicates substantial separation of the gene clusters shown in the genome. Genes of special interest are color coded, other genes of aromatic biosynthesis are shown in gray and all other genes are shown in white.

or from that present in lower-gamma Proteobacteria (based upon analysis of inter-domain linker regions; unpublished data).

Consensus ancestral gene organizations for the most densely represented divisions of Proteobacteria have been deduced as shown at the bottom of Fig. 5. Detailed information that supports a deduced consensus for ancestral gene organizations with respect to beta Proteobacteria, upper-gamma Proteobacteria, and lower-gamma Proteobacteria are shown later (Figs. 6, 7). We suggest that the last common ancestor of all Proteobacteria possessed the gene organization aro $Q \bullet p h e A>h i s H_{\mathrm{b}}>t y r A>\operatorname{aro} \mathrm{F}>\mathrm{cm} k>r p s A$. This is similar to the synteny that has been retained in general by the beta Proteobacteria and the upper-gamma Proteobacteria. The aroQ $\bullet$ pheA $>$ his $H_{\mathrm{b}}>t y r A$ portion likely specified all the catalytic requirements for conversion of chorismate to PHE and conversion of chorismate to TYR. 


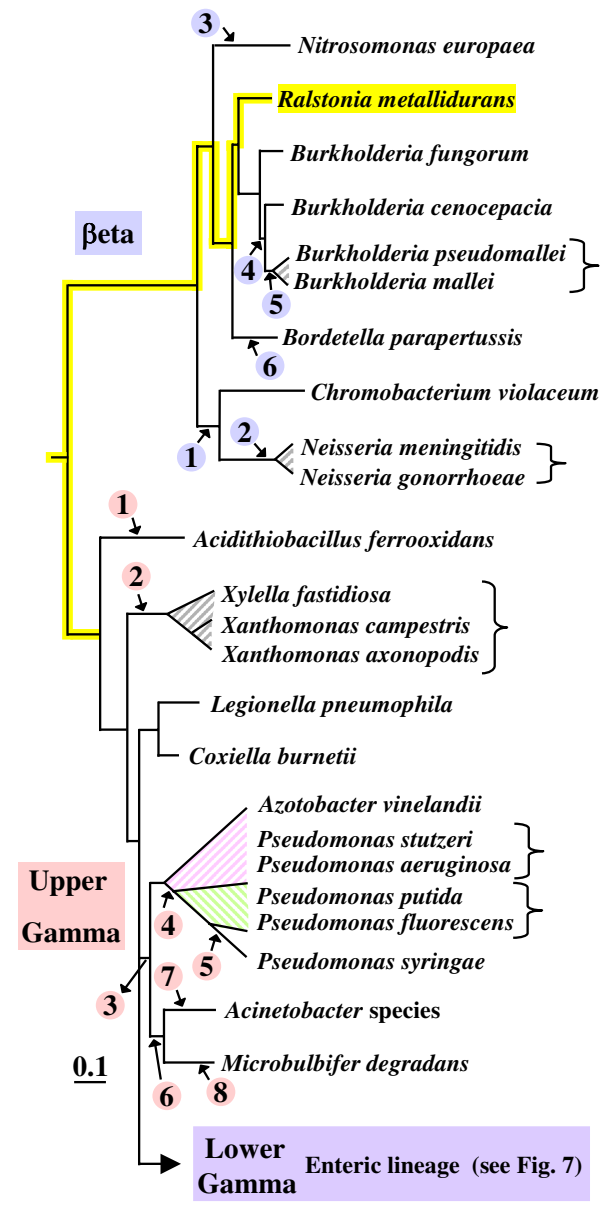

gyrA $>$ serC $>$ serA $>$ aroQ•pheA $>$ hisH $>$ tyrA $\quad$ aroF $>$ cmk $>$ rspA $>$ himD

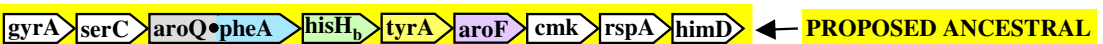
gyrA $>$ serC $>$ ORF $>$ aroQ॰pheA $>$ hisH $>$ tyrA $>$ aroF $>$ cmk $>$ rspA gyrA $>$ serC $>$ ORF $>$ aroQ॰pheA $>$ tyrA $>$ aroF $>$ cmk $>$ rspA $>$ himD

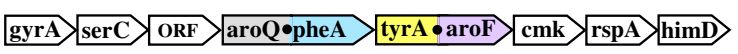

gyrA $>$ serC $>$ aroQ॰pheA $>$ tyrA $>$ aroF $>$ cmk $>$ rspA $>$ himD gyrA ORF serC tyrA $\quad$ aroF cmk

GENE DISPERSAL

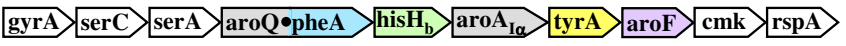

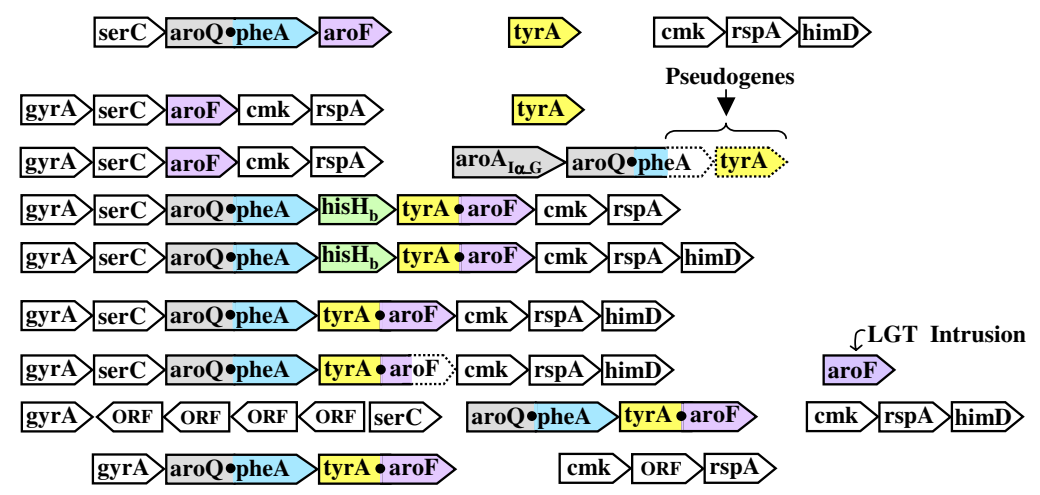

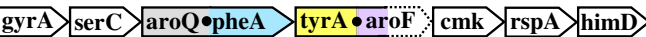

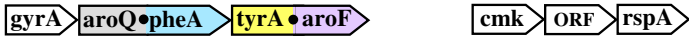
SYNTENY

\section{Figure 6}

Zoom-in from Fig. 5 showing tyrA synteny for the beta Proteobacteria and the upper-gamma Proteobacteria. The tree shown, based upon I6S rRNA sequences of the indicated organisms, indicates correct branching orders, but (to facilitate presentation) is not strictly correct in proportion. Circled numbers (in violet) indicate deduced evolutionary events for the beta Proteobacteria (see top of Table 5), whereas circled numbers (in pink; see bottom of Table 5) correspond to deduced evolutionary events for the upper-gamma Proteobacteria. Gene organizations of organisms indicated are shown on the right. The dotted outlining of some gene boxes in Coxiella burnetii and in P. syringae indicates pseudogene status.

Chorismate mutase activity specified by the aroQ domain could supply PPA for both PHE and TYR biosynthesis. Likewise, $\mathrm{HisH}_{\mathrm{b}}$, widely utilized as an aromatic aminotransferase [49], could also function for both PHE and TYR biosynthesis. Though currently available members of delta and epsilon Proteobacteria exhibit substantial gene scrambling, the various fragmentary linkage patterns seen provide support for the ancestor proposed. Geobacter (and other Delta_1 members) has the aroQ $\bullet$ pheA $>$ tyrA $>$ aroF $>c m k>r p s A$ linkage group (with $l y t B$ inserted between $c m k$ and $r p s A)$. Desulfovibrio vulgaris, another delta Proteobacterium (Delta_2) that is highly divergent from Geo- bacter, has a very interesting pattern of conservation and scrambling. aroQ $\bullet$ pheA $>$ aroF $>$ tyrA has been attached to a complete 7-gene trp operon. his $H_{\mathrm{b}}>c m k$ (not shown in Fig. 5) is completely separated from $r p s A$. The supraoperonic gene organization shown for $D$. vulgaris begins with two recently discovered genes, herein denoted aro $A^{\prime}$ and aroB', that encode enzymes specifying an alternative biochemical route to dehydroquinate [52]. The epsilon Proteobacteria all display significant gene scrambling, but piecemeal evidence for the unscrambled ancestor proposed is present. For example, Campylobacter jejuni possesses an aroQ $\bullet$ pheA $>$ his $H_{\mathrm{b}}$ unit, as well as $\operatorname{aroF}>l y t B>$ 

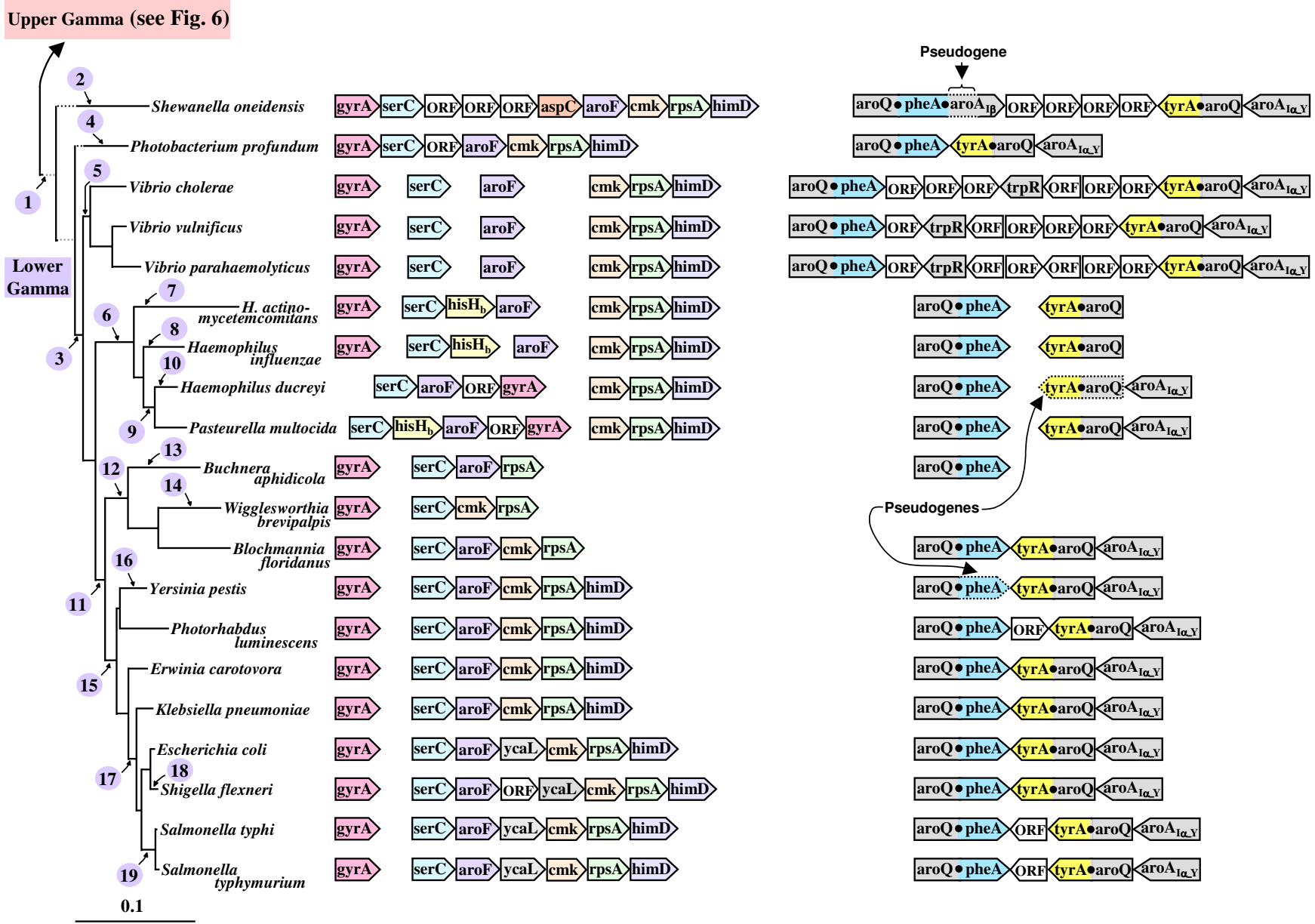

\section{Figure 7}

Zoom-in from Fig. 5 showing tyrA synteny for the lower-gamma Proteobacteria (enteric lineage). Deduced phylogenetic events numbered on the left are described in Table 6. The branching position for Buchnera is as suggested in ref. [7]. Dotted horizontal lines near the top of the tree indicate branch lengths that were shortened for convenience of presentation. Dotted outlining of boxes around some genes indicates their pseudogene status. It is unknown if the various open reading frame (ORF) insertions are functional.

$r p s A$ (Fig. 5). Wollinella succinogenes and Helicobacter hepaticus both possesses an $a r o F>l y t B>r p s A$ unit.

The ancestor of alpha Proteobacteria has lost the aroQ $\bullet$ pheA fusion, and a stand-alone pheA is consistently observed. Members of this group are quite uniform in the stable possession of $h i s H_{\mathrm{b}}>t y r A$ and $a r o F>c m k>r p s A$ as two separated linkage groups. The beta Proteobacteria are represented by members that have the gene organization: $\operatorname{ser} \mathrm{C}>\operatorname{aro} \mathrm{Q} \bullet p h e \mathrm{~A}>\operatorname{his} \mathrm{H}_{\mathrm{b}}>\operatorname{tyr} \mathrm{A}>\operatorname{aroF}>c m k>r p s A$. This is also seen in the members of the upper-gamma Proteobacteria.

Figure 5 includes organisms that illustrate the traces of synteny that can be detected in Bacteria where overall genome representation is just barely adequate. The following two figures illustrate how syntenic patterns of more resolution and refinement become evident with denser genome representation. 
Table 5: Key to evolutionary events asserted in Figure 6

\begin{tabular}{|c|c|c|}
\hline Group & & Evolutionary event(s) proposed \\
\hline \multirow[t]{6}{*}{ Beta } & I & $\begin{array}{l}\text { Dispersal of aroQ } \bullet p h e A>h i s H_{b}>t y r A \text { away from one another and away from } \operatorname{gyr} A>\operatorname{ser} C \text { and from } c m k>r s p A>h i m D \text {; } \\
\text { inversion of aroF with respect to } \mathrm{cmk} \text {. }\end{array}$ \\
\hline & 2 & Complete dispersal of all nine genes originally in the gyrA/himD linkage group. \\
\hline & 3 & Insertion of serA after serCa; separation of tyrA and aroF to yield the separated 6-gene unit and 4-gene unit shown. \\
\hline & 4 & Expulsion of his $\mathrm{H}_{\mathrm{b}}$ from the genome; insertion of 'ORF' after serC. \\
\hline & 5 & Fusion of tyrA with aroF. \\
\hline & 6 & Loss of $h i s H_{b}$ from genome. \\
\hline \multirow[t]{8}{*}{ Upper-Gamma } & I & Insertion of serA after serCa; insertion of $\operatorname{aro} A_{1 \alpha}$ after his $H_{b}$. \\
\hline & 2 & Translocation of his $\mathrm{H}_{b}$ and tyrA to other regions, leaving two separated 3-gene units. \\
\hline & 3 & Fusion of tyrA with aroF. \\
\hline & 4 & Loss of his $\mathrm{H}_{\mathrm{b}}$. \\
\hline & 5 & $\mathrm{~N}$-terminal deletion of $\bullet$ aroF domain, and acquisition of new aroF gene (probable LGT). \\
\hline & 6 & Separation of $c m k>r p s A>h i m D$ from aroQ $\cdot p h e A>t y r A \bullet a r o F$ \\
\hline & 7 & $\begin{array}{l}\text { Insertion of } 4 \text { unknown genes between gyrA and serC in opposite orientation and separation of gyrA > ORF > ORF > ORF } \\
>\text { serC from aroQ•pheA > tyrA•aroF. }\end{array}$ \\
\hline & 8 & Loss of himD; translocation of serC away from gyrA and aroQ•pheA. \\
\hline
\end{tabular}

aSince both Nitrosomonas (beta Proteobacteria) and Acidothiobacillus (upper-gamma Proteobacteria) emerge at deep positions in the tree of Fig. 5, an almost equally parsimonius possibility is that the ancestral serA was retained in this syntenic position in these two genera, but was transposed elsewhere shortly after early divergence.

\section{Zooming in on syntenic contexts of proteobacteria Beta proteobacteria and upper-gamma proteobacteria}

The beta Proteobacteria exhibit a dynamic but still interpretable pattern of altered synteny (Fig. 6 and Table 5). Species of Ralstonia have retained the proposed ancestral synteny that is marked with yellow highlighting in Fig. 6. This syntenic organization is such that the aromatic-gene unit $\operatorname{aroQ} \bullet p h e A>$ his $_{\mathrm{b}}>$ tyrA $>$ aroF is nested between gyrA $>\operatorname{ser} C$ at the leftward flank and $c m k>r p s A>\operatorname{himD}$ at the rightward flank. Species of Burkholderia (the next closest lineage) are almost identical, but exhibit individual evolutionary events (marked by circled numbers on the left, which correspond to a description of the proposed evolutionary events given in companion Table 5). These events include gene insertion, loss of $h i s H_{\mathrm{b}}$, translocation of genes away from the ancestral supraoperon, and fusion of tyr $A$ and $a r o F$ (in the common ancestor of $B$. mallei and B. pseudomallei). At a deeper level in the beta Proteobacteria section of the tree, Nitrosomonas europaea exhibits a separation of the ancestral supraoperon between tyrA and aroF. Either a very large insertion was made between tyrA and $\operatorname{aroF}$, or one of the two gene clusters shown was transposed as part of a sufficiently large segment to include all of the conserved flanking genes. In Chromobacterium violaceum tyrA has become completely isolated from other gene members of the ancestral supraoperon, and aroF has assumed an inverted orientation with respect to $\mathrm{cmk}$. Species of Neisseria exhibit no remnants of supraoperon synteny at all, and wholesale dispersal of all the supraoperon genes has occurred. (It is interesting that among the beta Proteobacteria, Neisseria species are also unique in that all of the trp-pathway genes are dispersed [7]).
The gamma Proteobacteria have separated into two distinctly different synteny patterns. The lower-gamma Proteobacteria have undergone marked syntenic change (see below). The assemblage portrayed between Acidithiobacillus and Microbulbifer in the lower part of Fig. 6 (termed the upper-gamma Proteobacteria) exhibit a strong overall syntenic resemblance of supraoperon genes to that of the beta Proteobacteria. Acidithiobacillus possesses a nearintact ancestral supraoperon, differing only in having two insertions: one gene encoding 3-deoxy-D-arabino-heptulosonate 7-phosphate (DAHP) synthase between his $H_{b}$ and $t y r A$, and the other being the insertion of ser $A$ between serC and aroQ•pheA. Pseudomonas aeruginosa and P. stutzeri have also retained nearly intact ancestral supraoperons, differing only in the fusion of tyrA and aroF. The serC $>$ aro $Q \bullet p h e A>h i s H_{\mathrm{b}}>\operatorname{tyr} A \bullet \operatorname{aroF}>c m k>r p s A$ supraoperon has been studied in $P$. stutzeri $[5,6]$. The tyrA $\bullet$ aroF fusion occurred in the common ancestor of the clade shown between Azotobacter and Microbulbifer in Fig. 6. The supraoperons of $P$. syringae, $P$. fluorescens and $P$. putida lack his $H_{\mathrm{b}}$. P. syringae exhibits a recent C-terminal truncation of the aroF domain, coupled with acquisition elsewhere in the genome of a free-standing $\bullet$ aroF that is not phylogenetically congruent (probably of LGT origin). Acinetobacter sp. and Microbulbifer degradans possess an aro $Q \bullet$ pheA $>$ tyrA $\bullet$ aroF unit that has become dissociated from serC at one end and from $\mathrm{cmk}$ on the other end. In Xylella and Xanthomonas, his $H_{b}$ has been deleted from the genome and tyrA has been transposed away from serC > aroQ $\bullet$ pheA $>$ aroF. The latter unit has been transposed away from gyrA, the ancestral flanking gene. On the other 
Table 6: Key to evolutionary events asserted in Figure 7

\begin{tabular}{|c|c|}
\hline Number & Evolutionary events proposed \\
\hline I & 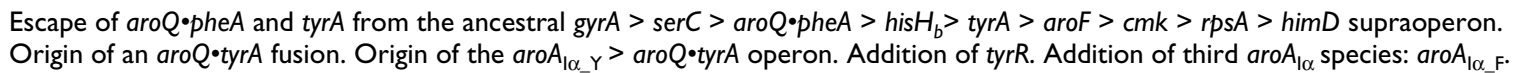 \\
\hline 2 & Fusion of $\operatorname{aro} Q \cdot p h e A$ with $a r o A_{1 \beta}$ pseudogene of unknown origin. Replacement of his $H_{b}$ by aspC duplicate linked with three ORFs. \\
\hline 3 & Dissociation of gyrA and serC. \\
\hline 4 & Removal of all genes intervening between aroQ•pheA and aroQ•tyrA. \\
\hline 5 & $\begin{array}{l}\text { Dissociation of } \operatorname{aroF} \text { from both } \operatorname{ser} C \text { and } c m k>\operatorname{rps} A>\operatorname{him} D \text {. Insertion of } \operatorname{trp} R \text { within the intervening region between aro } Q \cdot p h e A \text { and } \\
\text { aroQ } \operatorname{tyr} A \text {. }\end{array}$ \\
\hline 6 & Dissociation of $\operatorname{ser} C>$ his $H_{b}>\operatorname{aroF}$ from $c m k>r p s A>h i m D$ \\
\hline 7 & Loss of $a r o A_{1 \alpha \_Y}$ from tyr operon. \\
\hline 8 & aroF becomes dissociated from his $H_{b}$, and $a r o A_{I \alpha_{-} Y}$ is removed from the tyrA operon. \\
\hline 9 & $O R F>$ gyrA is inserted after aroF. \\
\hline 10 & aroQ•tyrA becomes a pseudogene. \\
\hline II & his $H_{b}$ is lost. \\
\hline 12 & himD is lost. \\
\hline 13 & $\mathrm{cmk}, \operatorname{himD}$ and $\operatorname{aro} A_{\mathrm{l \alpha} Y}>\mathrm{aro} Q \bullet t y r A$ are lost. \\
\hline 14 & $\operatorname{aroF}, \operatorname{himD}, \operatorname{aro} Q \bullet p h e A$, and $\operatorname{aro} A_{I \alpha_{Y} Y}>\operatorname{aro} Q \bullet t y r A$ are lost. \\
\hline 15 & All intervening genes between $\operatorname{aro} Q \bullet p h e A$ and $\operatorname{aro} Q \bullet t y r A$ are eliminated. \\
\hline 16 & pheA domain of aro $Q \bullet p h e A$ becomes a pseudogene. \\
\hline 17 & Insertion of ycaL between $a r o F$ and $\mathrm{cmk}$. \\
\hline 18 & Insertion of ORF between aroF and ycal. \\
\hline 19 & Insertion of ORF between aroQ•pheA and aro $Q \bullet t y r A$. \\
\hline
\end{tabular}

hand, $c m k>r p s A$ has remained next to himD, the gene usually flanking $r p s A$.

The enteric lineage

The lower-gamma Proteobacteria differ sharply from upper-gamma Proteobacteria in their possession of the $t_{y} A_{c_{-} \Delta}$ class of tyrA and its fusion with aroQ. In Fig. 2 this clade of AroQ $\bullet \mathrm{TyrA}_{\mathrm{c} \Delta}$ fusions was presented as one exhibiting absolute specificity for $\mathrm{NAD}^{+}$, combined with an overwhelming but not complete specificity for PPA. In Fig. 7 the gene synteny associated with $\operatorname{tyrA}_{c_{-} \Delta}$ is profiled against the 16S rRNA phylogenetic trees of the lowergamma Proteobacteria possessing these genes, and the proposed evolutionary events are summarized in the companion Table 6. Figure 5 has indicated a synteny consensus for the common ancestor at this hierarchical level whereby $g y r A>\operatorname{ser} C>$ his $H_{\mathrm{b}}>\operatorname{aroF}>c m k>r p s A$ parallels the ancestral synteny of $\beta$-Proteobacteria, but without aroQ $\bullet$ phe $A$ or tyrA in the middle of the linkage group. Many dynamic evolutionary events of altered aromatic biosynthesis have occurred within the lower-gamma Proteobacteria since their divergence from the upper-gamma Proteobacteria. This includes the emergence of three allosterically distinct DAHP synthases, one of which now comprises the two-gene, three-domain tyr operon $\left(\operatorname{aro} A_{\mathrm{I} \alpha \_Y}>\operatorname{aroQ} \bullet t y r A_{c_{\triangle} \Delta}\right)$. The upper-gamma Proteobacteria characteristically possess the $\operatorname{aro}_{I \alpha}$ paralogs encoding $\operatorname{AroA}_{\mathrm{I} \alpha_{-} \mathrm{H}}$ (TRP-inhibited DAHP synthase) and $\operatorname{AroA}_{\mathrm{I} \alpha_{-} \mathrm{Y}}$ (TYR-inhibited DAHP synthase). It has been asserted that
AroA $_{\text {I } \alpha \text { F }}$ (PHE-inhibited DAHP synthase) was the most recent paralog, acquired just after divergence of the lowergamma Proteobacteria [53]. It is bizarre that Shewanella oneidensis possesses a pseudogene of aro $A_{\mathrm{IB}}$ fused to the $\mathrm{C}$ terminus of aroQ $\bullet$ pheA. The $\operatorname{aro}_{\mathrm{I} \beta}$ subclass of Family-I DAHP synthases is not usually observed in gram-negative bacteria [54].

The dissociation of $t y r A_{c_{\Delta}}$ from the $\operatorname{ser} C / r p s A$ linkage group correlates with the fusion of $\operatorname{aro} Q$ with $t y r A_{c \_\Delta}$. The aroQ $\bullet$ pheA fusion has also escaped from the serC/rpsA linkage grouping and has become linked with the newly emerged tyr operon. Some sort of duplication and recom-

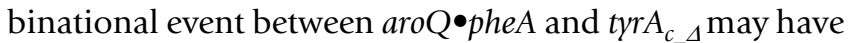
led to the creation of aroQ $\bullet$ tyr $A_{c_{\Delta} \Delta}$ since the AroQ $\bullet$ PheA proteins of lower-gamma Proteobacteria are distinct from AroQ $\bullet$ PheA proteins of other Proteobacteria with respect to the inter-domain linker length and the indel content (data not shown).

Although it usually is absent from the lower-gamma Proteobacteria, $\mathrm{HisH}_{\mathrm{b}}$ has persisted as the broad-specificity aromatic aminotransferase in the Pasteurella/Haemophilus grouping where two hisH paralogs are generally present, one of narrow specificity (denoted $h i s H_{n}$ ) being within the histidine operon. The aspC gene next to aroF in Shewanella is a paralog that probably functions as an aromatic aminotransferase, suggestive of the situation in the $E$. coli grouping where tyrB is a close paralog relative of as $p C$, tyrB 
having become specialized for aromatic biosynthesis [49]. Gene reduction associated with both endosymbiotic and pathogenic lifestyles are evident. Thus, Buchnera lacks tyr $A, c m k$, his $H, t y r B$, and possesses only a single $\operatorname{aro}_{I \alpha}$ species $\left(\operatorname{aro}_{\mathrm{I} \alpha H}\right)$. Haemophilus ducreyi also lacks tyrA, as well as aro $\mathrm{A}_{\mathrm{I} \_\mathrm{H}}$ and the entire trp operon [5].

\section{TyrA in its context of regulation TyrR regulon}

Knowledge of the gene regulation impacting TyrA in prokaryotes is sparse, being limited to the lower-gamma Proteobacteria. Here, extensive information gathered from $E$. coli has revealed that $\operatorname{aroQ} \bullet \operatorname{tyr} A_{c \_\Delta}$ belongs to a large regulon controlled by the TyrR repressor. The limited phylogenetic distribution of TyrR, being present only in the lower-gamma Proteobacteria (Fig. 8), indicates that it is a recent evolutionary acquisition. In E. coli the regulon members that are under the control of tyrR are the $a_{0 r o} A_{\mathrm{Id} \_} \mathrm{Y}$ $>$ tyrA operon, the aroLM operon, tyrP, tyrB, aroP, mtr, $\operatorname{aro} A_{\text {I } \alpha \mathrm{F}}$ and tyrR itself [55]. Thus, tyrR not only regulates the tyrosine branch of the pathway, but heavily impacts the common pathway and the transport of all three aromatic amino acids as well.

Although outside the scope of this study, a logical expansion of it would be to examine the individual evolutionary histories of all the members of the contemporary E. coli TyrR regulon, i.e., asking when and in what order did these genes come under the influence of tyrR? Clearly, the recruitment of structural genes by tyrR has been recent, quite dynamic and even now, exhibits evidence of further ongoing change. For example, tyrosine phenol-lyase (a catabolic enzyme that is only sparsely present in gamma Proteobacteria) has been recruited to the TyrR regulons of Erwinia herbicola [56] and Citrobacter freundii [57]. In these cases, not only does TyrR perform as a transcriptional activator, but it requires cyclic AMP receptor protein and integration host factor to do so.

As exemplified by E. coli, TyrR is generally a repressor. However, the transcriptional expression of $m t r$ is activated by TyrR in the presence of TYR, and tyrP is activated in the presence of PHE (although it is repressed in the presence of TYR). The N-terminal domain of TyrR has been associated with the ability of TyrR to activate transcription in the case of $m$ tr and tyrP [55]. Members of the Haemophilus/Pasteurella lineage have all lost the $\mathrm{N}$-terminal domain and presumably all lack the ability to accomplish transcriptional activation, as has been demonstrated experimentally with $H$. influenzae TyrR [58].

In view of the interesting complexity that two operons ( $m$ tr and aroLM) in E. coli are regulated by both tyrR and $\operatorname{trpR}$ [55], it may be more than coincidental that $t y r R$ and trpR seem to have emerged at about the same evolutionary time, i.e., coincident with the divergence of the uppergamma Proteobacteria from the lower-gamma Proteobacteria (Fig. 7). A possible interaction between the TyrR and TrpR proteins has been noted [55].

\section{PhhR in relationship to aromatic catabolism}

Arias-Barrau et al. [59] have recently characterized a central catabolic pathway (Hmg) that degrades homogentisate in three steps to fumarate and acetoacetate as a source of carbon and energy. One of several peripheral pathways feeding into the central pathway begins with PHE and produces homogentisate via the reactions of phenylalanine hydroxylase (Phh), aromatic aminotransferase, and 4-hydroxyphenylpyruvate dioxygenase (Hpd). In the absence of Phh, a shorter version of the peripheral pathway is one that can use TYR, but not PHE, as a source of carbon and energy. In Fig. 8 the presence of Phh, Hpd, and Hmg segments of catabolism are mapped on a $16 \mathrm{~S}$ rRNA tree. (The aromatic aminotransferase distribution is not shown since a multiplicity of aromatic aminotransferases having overlapping substrate specificities makes it particularly challenging to identify the functional role [49].) The cyanobacteria are unique among Bacteria in the use of Hpd for a completely different metabolic role unrelated to aromatic catabolism, i.e., the synthesis of vitamin E derivatives [60].

PhhR is a homolog of TyrR that has been shown in P. aeruginosa to be a divergently transcribed activator of a 3gene operon needed for PHE and TYR catabolism [61]. The structural genes encode phenylalanine hydroxylase ( $p h h A)$, carbinolamine dehydratase ( $p h h B)$ and 4-hydroxyphenylpyruvate aminotransferase $(p h h C)$, and are powered by a $\sigma^{54}$ promoter $[61,62]$. PhhR evolved relatively recently since it is only present in some gamma Proteobacteria (Fig. 8). The ancestral regulatory gene for the Phh peripheral pathway may have been a member of the leucine-responsive regulatory protein/asparagine synthase $\mathrm{C}$ (Lrp/AsnC) family judging from the adjacent and divergently oriented position of asnC genes to phhA in organisms such as Xanthomonas axonopodis and Mesorhizobium loti. A recent overview of the many different regulator families involved in the control of aromatic catabolism conveys an emerging sense of the variety and dynamic evolutionary processes that underlie aromatic catabolism [63]. Occasional distant homologs of phhR that appear in erratic fashion (see Fig. 9) may have some other regulatory function. For example, Clostridium tetani may use its PhhR homolog as a transcriptional activator of the gene encoding tyrosine phenol-lyase, as occurs in species of Erwinia [56] and Citrobacter [57].

\section{Relationship of TyrR and PhhR}

What might be of origin of TyrR? TyrR is an anomalous member of the large prokaryote family of $\sigma^{54}$ enhancer- 


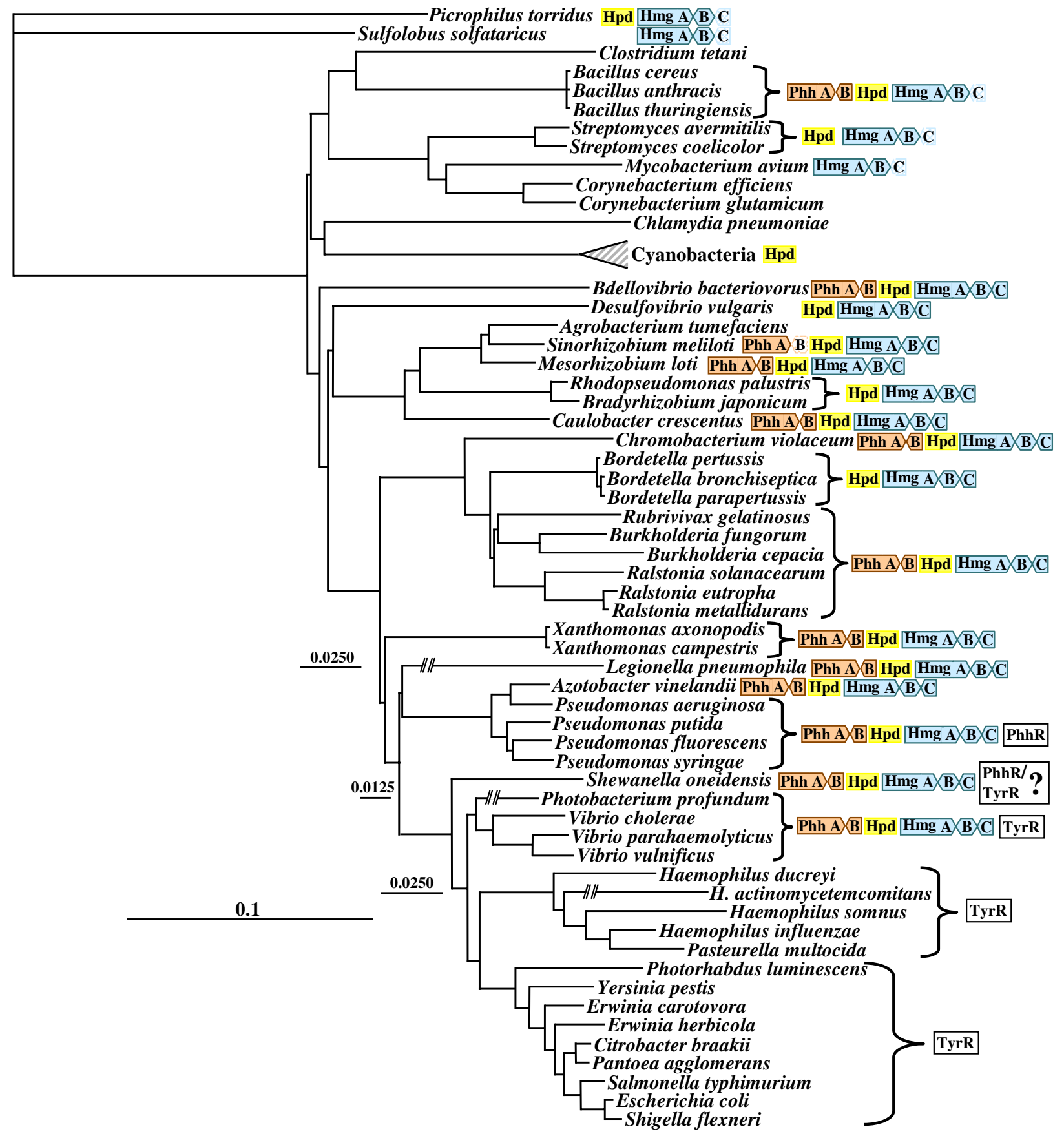

Figure 8

Distribution of modules of aromatic catabolism mapped on a 16S rRNA tree. In this figure, only presence or absence (not gene order) is indicated. The Phh module (orange) consists of phenylalanine hydroxylase (PhhA), carbinolamine dehydratase (PhhB), and tyrosine aminotransferase (not shown, see Text), and accomplishes the overall conversion of PHE to 4-hydroxyphenylpyruvate. The Hpd module (yellow) is 4-hydroxyphenylpyruvate dioxygenase, which converts 4-hydroxyphenylpyruvate to homogentisate. The Hmg module (blue) catalyzes the 3-step conversion of homogentisate to acetoacetate and fumarate. The distribution of PhhR and TyrR is shown in boxes. In some cases the HmgC member is shaded light blue to indicate that the gene encoding this isomerase could not be found and is probably encoded by an as yet unknown analog. Some long branches are drawn with gaps that represent $25 \%$ of the length of the scale bar. 

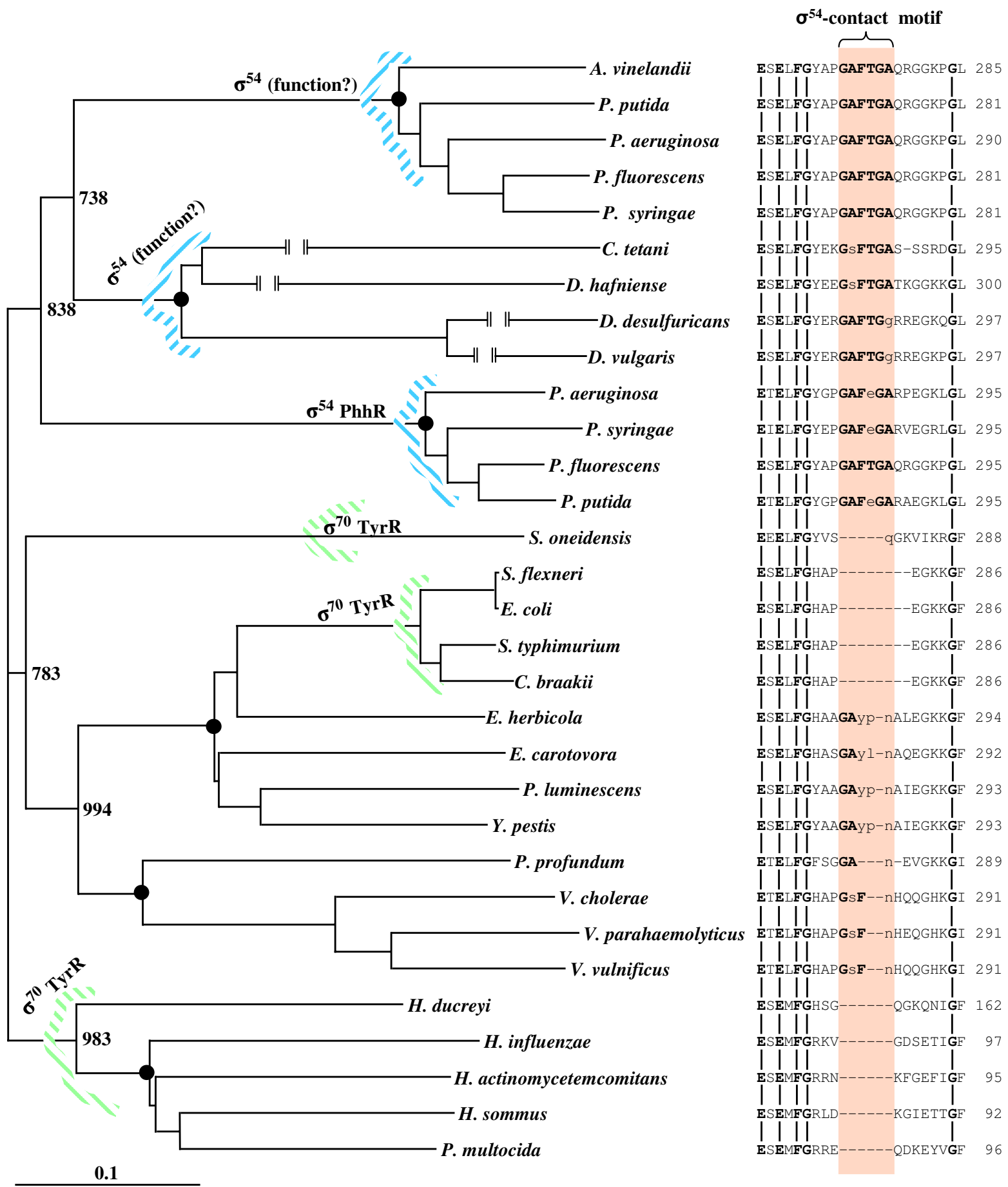

Figure 9

Protein tree of TyrR homologs. Nodes supported by bootstrap values of 998 or more are marked with solid circles, and the bootstrap values for nodes internal to these are shown. Generic names relevant to the organism abbreviations can be viewed in Fig. 8. A conserved region containing the $\sigma^{54}$ contact motif GAFTGA is highlighted as an orange band. "Imperfect" residues in this region are shown in lower-case fonts. Residue numbers are shown at the right. TyrR and PhhR are regulators of $\sigma^{70}$ and $\sigma^{54}$ promoters, respectively. Four $\sigma^{54}$ proteins of unknown function have very long branches, and to facilitate the visual presentation, the gaps in branch continuity shown represent a scale-bar distance of 0 . I. Clades possessing $\sigma^{54}$ regulators are indicated with blue stripes, and $\sigma^{70}$ regulators are indicated with green stripes. 
binding proteins that activate promoters dependent upon $\sigma^{54}$. TyrR is unique within its homology grouping in that it targets $\sigma^{70}$ promoters for regulation, usually (but not always) being a repressor. Its closest homolog relative is PhhR, a canonical member of $\sigma^{54}$ enhancer-binding proteins. $\sigma^{54}$-dependent enhancer proteins possess a highly conserved $\sigma^{54}$-contact motif, GAFTGA, that is intimately involved in formation of the ternary complex of enhancer and $\sigma^{54}$-RNA polymerase holoenzyme [64]. This is perfectly or nearly perfectly retained in the upper clades shown in Fig. 9, but is disrupted or completely absent in the clades between Shewanella oneidensis and Pasteurella multocida. The deeper phylogenetic distribution of PhhR (Fig. 8) suggests that TyrR evolved as a variant of PhhR. If correct, a regulatory gene that is oriented to catabolism $(p h h R)$, and itself of relatively recent origin, was conscripted even more recently for a completely new role in the regulation of primary biosynthesis (tyrR).

Consistent with the latter supposition, the gain of TyrR generally correlates with the loss of competence for aromatic catabolism (Fig. 8). In contrast to the Citrobacter/ Salmonella/Escherichia/Shigella and the Pasteurella/Haemophilus clades (whose TyrR homologs completely lack the GAFTGA motif), the remaining enteric clades have retained some residues in this region. These residues appear to be more than random remnants. It would be interesting to know if these residues have any functional significance. Indeed, the Photobacterium/Vibrio clade has retained the ancestral catabolic capabilities (Fig. 8) that would appear to demand retention of regulation via PhhR; yet the parallelism of the overall features of biosynthesis that are shared with other lower-gamma Proteobacteria would seem, on the other hand, to demand TyrRmediated regulation. Perhaps this "TyrR" species participates in the regulation of both catabolic and biosynthetic genes. In this connection, it is interesting that Chaney et al. [64] found that a change in the GAFTGA motif of NifA could be partially "suppressed" by mutational changes in the $\mathrm{N}$-terminal region of $\sigma^{54}$.

Even more striking as a possible evolutionary intermediate is the most outlying member of the lower- gamma Proteobacteria, Shewanella oneidensis. The position of its TyrR on the protein tree parallels expectations based on the $16 \mathrm{~S}$ rRNA tree. This, plus the conservation of the TyrR regulon features and the overall gene synteny suggest $E$. coli-like function as TyrR, i.e. acting as a general repressor of regulon-member $\sigma^{70}$ promoters engaged in aromatic biosynthesis. However, the location of "tyrR" in $S$. oneidensis between $p h h A$ and $p h h B$ on one side, and $h m g B$ and $h m g C$ on the other side, strongly implies some kind of regulatory relationship with the catabolic genes. It would be quite interesting to determine experimentally whether "TyrR" in S. oneidensis (and maybe Vibrio, as well) can function as a repressor of the usual suite of $\sigma^{70}$ promoters, as well as an activator of $\sigma^{54}$ promoters for $p h h A / p h h B$ and/or $h m g B / h m g C$.

We suggest that TyrR evolved as a modified version of PhhR as follows. In view of the distribution of genes encoding PhhR and TyrR, as well as the aforementioned catabolic enzymes, the most parsimonious evolutionary scenario may be that central and peripheral catabolic pathways depicted in Fig. 8 are quite ancient, but acquisition of PhhR as a $\sigma^{54}$-dependent activator of phenylalanine hydroxylase was quite recent, originating about the time of divergence of gamma Proteobacteria. The clade defined by Shewanella/Vibrio/Photobacterium retained the catabolic pathway, whereas the other enteric lineages discarded the catabolic pathway, but retained PhhR, which was then recruited as a $\sigma^{70}$-dependent regulator of aromatic biosynthesis (TyrR).

\section{Regulation by attenuation}

A widespread mechanism of regulation is via an attenuation mechanism whereby transcripts initiated at given promoters can be terminated prior to reaching the structural genes of an operon. Whether termination occurs usually depends on the balance (dictated by a variety of mechanisms) between mutually exclusive terminator and anti-terminator structures [65].

Merino has developed a website [66] to provide a database of putative attenuators ahead of various operons in Bacteria. We screened this database for likely attenuators relevant to the regulation of tyrA. Table 7 shows intriguing results that point to significant experimental work that would be desirable. tyrA is frequently a member of apparent supraoperons, as alluded to elsewhere in this paper, and some of these appear to be large gene clusters controlled by attenuation. Substantial work is needed to establish the depth of clades possessing a given attenuator. For example, the $h i s H_{b}>$ tyrA operon is reliably present throughout all alpha Proteobacteria. Since Agrobacterium tumefaciens has been found to possess an attenuator ahead of the his $H_{b}>$ tyrA operon, one might reasonably expect that most of the alpha Proteobacteria would possess the attenuator as well. If not, this attenuator would have been a very recent evolutionary innovation. Likewise, since the $a r o A_{I \alpha \_Y}>t y r A$ operon is widely present throughout the lower-gamma Proteobacteria, it would be interesting to confirm whether only the several species of Vibrio identified on the Merino website have an attenuator ahead of this operon (or whether other attenuators present are too weak to exceed the threshold imposed for preliminary detection).

Some of the supraoperons that appear to be controlled by attenuation are interesting in that they contain the 
Table 7: Putative attenuators ${ }^{\mathrm{a}}$ associated with tyrA

\begin{tabular}{|c|c|c|}
\hline Organism & Gene organization $^{\mathrm{b}}$ & Fig $^{\mathrm{c}}$ \\
\hline Agrobacterium tumefaciens & $\neg$ his $H_{b}>$ tyrA & $4^{d}$ \\
\hline Bacillus anthracis & ᄀaroG > his $\mathrm{H}_{b}>$ tyrA $>$ aroF & (II) \\
\hline Bacillus cereus & ᄀaroG $>$ his $H_{b}>$ tyrA $>$ aroF & (II) \\
\hline Bacillus halodurans & $\neg$ tyrA $>$ aroF & (II) \\
\hline Bacteroides thetaiotaomicron & $\neg$ phe $A>$ his $_{b}>\operatorname{aroA}_{l} \beta^{\bullet a r o Q}>\operatorname{tyrA}$ & 4 \\
\hline Bordetella parapertussis & $\neg$ gyrA $>\operatorname{ser} C>$ aro $Q \cdot p h e A>\operatorname{tyr} A>\operatorname{aroF}>\mathrm{cm} k>\operatorname{rps} A>\operatorname{him} D$ & 5 \\
\hline Desulfovibrio vulgaris & ᄀaroA' $>$ aro $B^{\prime}>\operatorname{aro} Q \cdot p h e A>\operatorname{aroF}>\operatorname{tyr} A>$ [trp operon] & 4 \\
\hline Enterococcus faecalis & $\neg \operatorname{aro} D>\operatorname{aro}_{l \beta}>\operatorname{aro} B>\operatorname{aro} G>\operatorname{tyr} A>\operatorname{aroF}>\operatorname{aro} E>$ pheA & 4 \\
\hline Lactococcus lactis & ᄀysaA $>$ blrG > kinG $>$ tyrA $>$ aroF $>$ aroE $>$ phe $A$ & 4 \\
\hline Lactobacillus plantarum & $\neg \mathrm{ORF}>$ aroG $>\mathrm{ORF}>\operatorname{aroF}>\operatorname{tyr} A>\operatorname{aroE}$ & \\
\hline Listeria innocua & $\neg \operatorname{aroG}>\operatorname{aroB}>\operatorname{aroH}>$ hisH $_{b}>\operatorname{tyrA}>\operatorname{aroF}$ & $4,(11)$ \\
\hline Streptococcus pneumoniae & $\neg O R F>a r o C_{1}>a r o D>a r o B>a r o G>$ tyrA $>\neg O R F>a r o F>a r o E>p h e A$ & 4 \\
\hline Thermoanaerobacter tencongensis & $\neg$ phe $A>$ aro $A_{1 \beta}>$ tyrA $>$ aroF $>$ ORF $>$ ORF & \\
\hline Thermus thermophilus & ᄀaroA $A_{l \beta}>\operatorname{tyrA}$ & \\
\hline Vibrio parahaemolyticus & $\neg \operatorname{aro} A_{l \alpha Y}>$ tyrA & 6 \\
\hline Vibrio vulnificus & $\neg \operatorname{aro} A_{\mid \alpha_{-} Y}>$ tyrA & 6 \\
\hline
\end{tabular}

aAttenuators were extracted from the website of Merino [66]. Links are provided for viewing the complete data, including a visualization of the putative attenuator structures.

bThe symbol $\urcorner$ is used for attenuators. Genes encoding the alternative biochemical steps that were recently reported for formation of dehydroquinate from aspartate semialdehyde and ketohexose I-phosphate [52] are designated aroA' and aroB'.

CRefers to figures within this manuscript or, if enclosed within parentheses, to the figure in ref. [7].

dSee the consensus gene organization for $\alpha$ Proteobacteria.

majority of genes needed for both PHE and TYR biosynthesis, e.g., the supraoperons in Enterococcus faecalis and Streptococcus pneumoniae. The latter organism displays two attenuator units. The supraoperon of Desulfovibrio vulgaris is novel in that it begins with two relatively rare genes encoding alternative enzyme steps for aromatic biosynthesis [52], denoted here as aroA' and aroB'. The leading five genes are adjacent to the seven-gene $\operatorname{trp}$ operon.

\section{Conclusion}

Protein divergence within a vertical genealogy is not necessarily smooth and progressive. Qualitative biochemical innovations can result in a barrage of new selective pressures that result in evolutionary jumps. The consequent incongruence might easily be mistaken for LGT. The basis for evolutionary jumps will usually only be recognized by detailed and comprehensive analyses of any given subsystem. Examples in this study are as follows. (i) The tyr $A_{c \_\Delta}$ gene of the lower-gamma Proteobacteria has diverged markedly from $t y r A_{c}$ of the upper-gamma Proteobacteria. Here the milestone event was fusion of aroQ to a putative $\operatorname{tyrA}_{\mathrm{c}}$ in the ancestor of lower-gamma Proteobacteria to produce $\operatorname{aro} Q \bullet t y r A_{c_{\_} \Delta}$. Indels within the $\bullet$ tyr $A_{c_{\_} \Delta}$ domain presumably reflect a multiplicity of selections for functional interactions known to exist between the two fused domains as discussed earlier. (ii) Members of the subclass taxon Actinobacteridae possess $\mathrm{TyrA}_{\mathrm{a}}$ proteins that separate into two distinct groupings. The presumed ancestral
$\operatorname{NAD}(\mathrm{p}) \mathrm{TyrA}_{\mathrm{a}}$ that is still present in the Actinobacteridae_1 clade very likely spawned the divergent $\mathrm{NAD}^{+}$-specific variety of $\operatorname{TyrA}_{\mathrm{a}}$ to yield the contemporary Actinobacteridae_2 clade.

The previous evolutionary analysis of trp-pathway genes $[7,8]$ can be viewed as a model for comparable studies with other gene systems. Expansion to the greater aromatic pathway is a logical extension. The dynamics of evolutionary change for tyrA can be matched to the dynamics exhibited by the trp system. For example, the lower-gamma Proteobacteria separate as a distinct phylogenetic unit from beta Proteobacteria and upper-gamma Proteobacteria on criteria defined by milestone evolutionary events that altered many character states of both tryptophan and tyrosine biosynthesis in the lower-gamma Proteobacteria. In the future one can anticipate that comprehensive and systematic phylogenetic analysis of each protein member of the TYR, PHE and TRP branches, the common aromatic-pathway trunk, and minor vitamin-like branches (such as the 4-aminobenzoate/folate branch) will accommodate a progressively integrated picture of the entire aromatic network, including catabolic pathways and many other specialized pathways.

\section{Methods \\ TyrA sequences}

Most TyrA sequences were obtained from the National Center for Biotechnology Information (NCBI) [16]. TyrA 
sequences from incomplete genomes were retrieved from the PEDANT database [67]. Several sequences in our curated TyrA collection have been corrected for incorrect translation start sites. Various curated TyrA sequence files can be downloaded from our website. These files include complete sequences, trimmed catalytic-core domains, and amino-acid sequence segments that are relevant to specificity for pyridine nucleotide or to specificity for the cyclohexadienyl substrate. The sequence files are summarized in Table 3.

\section{Congruency groupings}

TyrA proteins that cluster together on the TyrA protein tree in congruence with the 16S rRNA tree are called congruency groups. Exact correspondence of branching orders is not necessarily observed. So far, congruency groupings have been assembled for tryptophan-protein concatenates [8] and for TyrA proteins. Completion of equivalent work with the remaining aromatic-pathway segments will identify the repertoire of bacterial organisms in possession of a "pure" vertical genealogy with respect to aromatic biosynthesis. Congruency groups for TyrA can be accessed at our AroPath website [9], where a listing of the membership of congruency groups is maintained and updated. Any members of congruency-group clusters, whose position there is incongruent with 16S rRNA expectations, probably (but not necessarily) originated by LGT. The donor lineage may not be obvious, but as more genomes come on line, many cases where donor identities are currently unknown may become revealed. A listing of "orphan" TyrA proteins that belong to no current congruency group is given. Such orphans reflect the lack of sufficient genome representation in particular phylogenetic regions and undoubtedly will become the nucleus for additional congruency groups in due course.

\section{Alignments}

Multiple alignments were obtained by use of the ClustalW or ClustalX programs (Version 1.83) [68]. Manual adjustments were needed in the region of the GxGxxG motif for binding of pyridine nucleotide cofactor in the $\mathrm{N}$-terminal region of TyrA proteins. Guidance for alignment was assisted by maximizing conformation with the Wierenga fingerprint, making allowance for a variable loop of 2-5 residues [26]. This was done with the assistance of the BioEdit multiple alignment tool of Hall (5.0.9 Edition) [69]. The refined multiple alignment was used as input for generation of a phylogenetic tree using the phylogeny inference package (Version 3.2), PHYLIP [70]. The neighbor-joining program was used to obtain a distance-based tree. The distance matrix was obtained by use of Protdist with a Dayhoff Pam matrix. The Seqboot and Consense programs were then applied to assess the statistical support of the tree using bootstrap resampling $(1,000$ replications). We also used the ANCESCON package [71], which produced similar results as shown in Fig. 2 (albeit with even wider separation of many groups). The presence of regulatory domains (ACT and REG) was accepted when indicated by the Domain Architecture Retrieval Tool (DART) on the BLAST menu at NCBI [16].

\section{Profile HMMs}

Profile hidden Markov models for each of the four TyrA subfamilies, $\operatorname{TyrA}_{\mathrm{a}^{\prime}}, \operatorname{TyrA}_{c^{\prime}}, \operatorname{TyrA}_{\mathrm{p}}$ and $t y r A_{c_{-} \Delta^{\prime}}$ were built using Sean Eddy's HMMER package [72]. The HMMs were generated from our file of curated cyclohexadienyl-substrate core segments (see Table 3 ). The seed sequences for each subfamily were first aligned using ClustalW [68]. The resulting multiple sequence alignments were then manually edited to produce more accurate alignment of the seed sequences. Finally, the edited multiple sequence alignments were used to generate the profile HMMs for each TyrA subfamily.

\section{Appraisal of gene fusions as one-time or multiple events}

Whether any given contemporary gene fusions tracked back to a fusion event in a common ancestor or whether they occurred independently was evaluated by phylogenetic analysis of the individual protein domains and by inspection of the inter-domain linker region. Linker regions were determined by multiple alignments of fusion sequences with corresponding free-standing domains present in the closest relatives to organisms that lack the gene fusions.

\section{Authors' contributions}

JS and MW integrated this specific effort with the broader and ongoing objective of implementing a dynamic and progressively updateable website (AroPath). JS also made substantial contributions to the bioinformatic work. CB did all of the art work and a majority of the bioinformatic analyses. RJ provided initial guiding concepts, a general organizational overview, and assembled the initial manuscript draft. $\mathrm{CB}, \mathrm{RJ}$, and JS contributed to the formulation of conclusions made, and all of the authors read and approved the final version of the manuscript.

\section{Additional material}

\section{Additional File 1}

Table S1, entitled "Key to organism acronyms and sequence identifiers", is provided as supplementary material in an html document. This table contains the full collection of sequence data and annotations contained in this paper, and gene identification (gi) numbers are included and hyperlinked to facilitate access to the corresponding GenBank records. For future reference to a progressively updated table, refer to the AroPath website [73].

Click here for file

[http://www.biomedcentral.com/content/supplementary/17417007-3-13-S1.html] 


\section{Acknowledgements}

R. Jensen thanks the National Library of Medicine (Grant GI3 LM008297) for partial support. This research is partially supported by the U. S. Army Research Institute of Infectious Diseases (USAMRIID). This analysis would not have been possible were it not for the yeoman efforts in comparative enzymology carried out over a period of more than 25 years by many students and postdoctoral fellows, most notably Graham S. Byng, Robert Whitaker, Alan X. Berry and Suhail Ahmad. This has produced an invaluable resource of comprehensive data, some of it unpublished. This paper is dedicated to our colleague and collaborator, John E. Gander, on the occasion of his $80^{\text {th }}$ birthday.

\section{References}

I. Xie G, Bonner CA, Jensen RA: Cyclohexadienyl dehydrogenase from Pseudomonas stutzeri exemplifies a widespread type of tyrosine-pathway dehydrogenase in the TyrA protein family. Comp Biochem Physiol C Toxicol Pharmacol 2000, 1 25:65-83.

2. Jensen RA: Tyrosine and phenylalanine biosynthesis: relationship between alternative pathways, regulation and subcellular location. Rec Adv Phytochem 1986, 20:57-82.

3. Todd $A E$, Orengo CA, Thornton JM: Evolution of function in protein superfamilies, from a structural perspective. J Mol Biol 200I, 307: IIII3-III43.

4. Teichmann SA, Rison SCG, Thornton JM, Riley M, Gough J, Clothia C: The evolution and structural anatomy of the small molecule metabolic pathways in Escherichia coli. J Mol Biol 200I, 3 I I:693-708.

5. Xie G, Brettin T, Bonner CA, Jensen RA: Mixed-function supraoperons that exhibit overall conservation, albeit shuffled gene organization, across wide intergenomic distances within eubacteria. Microb Comp Genomics 1999, 4:5-28.

6. Xie G, Bonner CA, Jensen RA: A probable mixed-function supraoperon in Pseudomonas exhibits gene organization features of both intergenomic conservation and gene shuffling. J Mol Evol 1999, 49:108-121.

7. Xie G, Keyhani N, Bonner CA, Jensen RA: Ancient origin of the tryptophan operon and the dynamics of evolutionary change. Microbiol Mol Biol Rev 2003, 67:303-342.

8. Xie G, Bonner CA, Song J, Keyhani NO, Jensen RA: Inter-genomic displacement via lateral gene transfer of bacterial trp operons in an overall context of vertical genealogy. BMC Biology 2004, 2: 15.

9. AroPath [http://AroPath.lanl.gov/Phylogeny/CG/tyrCG.html]

10. Gil R, Silva FJ, Zientz E, Delmotte F, Gonzalez-Candelas F, Latorre A, Rausell C, Kamerbeek J, Gadau J, Holldobler B, et al.: The genome sequence of Blochmannia floridanus : comparative analysis of reduced genomes. Proc Natl Acad Sci USA 2003, 100:9388-9393.

II. Gevers D, Vandepoole K, Simillion C, Van de Pere Y: Gene duplication and biased functional retention of paralogs in bacterial genomes. Trends Microbiol 2004, I 2: I 48- 154.

12. AroPath [http://AroPath.lanl.gov/Phylogeny/CG/index.html]

13. Blanc V, Gil P, Bamasjacques N, Lorenzon S, Zagorec M, Schleuniger $\mathrm{J}$ : Identification and analysis of genes from Streptomyces pristinaespiralis encoding enzymes involved in the biosynthesis of the 4-dimethylamino-L-phenylalanine precursor of pristinamycin I. Mol Microbiol 1997, 23:191-202.

14. Lingens F, Göbel W, Üsseler H: Regulation der biosynthesis der aromatischen aminosauren in Saccharomyces cerevisiae, I. Hemmung der Enzymaktivitaten (Feedback-Wirkung). Biochem Z 1966, 346:357-67.

15. Zamir LO, Jung E, Jensen RA: Co-accumulation of prephenate $L-$ arogenate and spiro-arogenate in a mutant of Neurospora. 1983, 258:6492-6496.

16. National Center for Biotechnology Information [http:// www.ncbi.nlm.nih.gov]

17. Xia T, Jensen RA: A single cyclohexadienyl dehydrogenase specifies the prephenate dehydrogenase and arogenate dehydrogenase components of the dual pathways to L-tyrosine in Pseudomonas aeruginosa. J Biol Chem 1990, 265:20033-20036.

18. Zhao G, Xia T, Ingram L, Jensen RA: An allosterically insensitive class of cyclohexadienyl dehydrogenase from Zymomonas mobilis. EurJ Biochem 1993, 212:157-165.
19. Jensen RA: Enzyme recruitment in evolution of new function. Annu Rev Microbiol 1976, 30:409-425.

20. Hall GC, Flick MB, Gherna RL, Jensen RA: Biochemical diversity for biosynthesis of aromatic amino acids among the cyanobacteria. J Bacteriol 1982, 149:65-78.

21. Subramaniam P, Bhatnagar R, Hooper A, Jensen RA: The dynamic progression of evolved character states for aromatic amino acid biosynthesis in gram-negative bacteria. Microbiology 1994, I 40:343I-3440.

22. Byng GS, Whitaker RJ, Gherna RL, Jensen RA: Variable enzymological patterning in tyrosine biosynthesis as a means of determining natural relatedness among the Pseudomonadaceae. J Bacteriol 1980, I 44:247-257.

23. Keller B, Keller E, Gorisch H, Lingens F: Biosynthesis of phenylalanine and tyrosine in Streptomycetes. Hoppe Seylers Z Physiol Chem 1983, 364:455-459.

24. Keller B, Keller E, Lingens F: Arogenate dehydrogenase from Streptomyces phaeochromogenes. Purification and properties. Biol Chem Hoppe Seyler 1985, 366: I063-1066.

25. Bonner CA, Jensen RA, Gander JE, Kehani NO: A core catalytic domain of the TyrA protein family: arogenate dehydrogenase from Synechocystis. Biochem J 2004, 382:279-29I.

26. Wierenga RK, Terpstra $P$, Hol WG]: Prediction of the occurrence of the ADP-binding $\beta \alpha \beta$-fold in proteins, using an amino-acid sequence fingerprint. J Mol Biol 1986, 187:10I-107.

27. Rippert $P$, Matringe M: Molecular and biochemical characterization of an Arabidopsis thaliana arogenate dehydrogenase with two highly similar and active protein domains. Plant Mol Biol 2002, 48:36I-368.

28. Rippert P, Matringe M: Purification and kinetic analysis of the two recombinant arogenate dehydrogenase isoforms of Arabidopsis thaliana. Eur J Biochem 2002, 269:4753-476I.

29. Xie G, Forst C, Bonner CA, Jensen RA: Significance of two distinct types of tryptophan synthase beta chain in Bacteria, Archaea and higher plants. Genome Biol 2002, 3:Research0004.1-0004.13.

30. Champney WS, Jensen RA: The enzymology of prephenate dehydrogenase in Bacillus subtilis. J Biol Chem 1970, 245:3763-3770.

3I. Xie G, Bonner CA, Brettin T, Gottardo R, Keyhani NO, Jensen RA: Lateral gene transfer and ancient paralogy of operons containing redundant copies of tryptophan-pathway genes in Xylella species and heterocystous cyanobacteria. Genome Biol 2003, 4:RI4.

32. Chen S, Vincent S, Wilson DB, Ganem B: Mapping of chorismate mutase and prephenate dehydrogenase domains in the Escherichia coli T-protein. Eur J Biochem 2003, 270:757-763.

33. Mavrodi DV, Ksenzenko VM, Bonsall RF, Cook RJ, Boronin AM, Thomashow LS: A seven-gene locus for synthesis of phenazine-Icarboxylic acid by Pseudomonas fluorescens 2-79. J Bacteriol 1998, I 80:254|-2548.

34. Pierson LS, Gaffney T, Lamb S, Gong F: Molecular analysis of genes encoding phenazine biosynthesis in the biological control bacterium. Pseudomonas aureofaciens 30-84. FEMS Lett 1995, 134:299-307.

35. AroPath [http://AroPath.lanl.gov/Annotation/CuratedAASeqFor Download.html]

36. Pfam [http://www.sanger.ac.uk/Software/Pfam/]

37. Interpro [http://www.ebi.ac.uk/interpro/]

38. Eddy SR: Profile-hidden Markov models. Bioinformatics 1998, 14:755-763.

39. Park J, Kaplus K, Barrett C, Hughey R, Haussler D, Hubbard T, Chothia $C$ : Sequence comparisons using multiple sequences detect three times as many remote homologues as pairwise methods. J Mol Biol 1998, 284: I20I-I2I0.

40. AroPath [http://AroPath.lanl.gov/Biosynthesis/TyrPath/hmmPfam TyrA.html]

41. AroPath [http://AroPath.lanl.gov/Biosynthesis/TyrPath/index.html]

42. Fazel A, Jensen R: Obligatory biosynthesis of $L$-tyrosine via the pretyrosine branchlet in coryneform bacteria. J Bacteriol 1979 , 138:805-8I5.

43. Fazel AM, Bowen JR, Jensen RA: Arogenate (pretyrosine) is an obligatory intermediate of $L$-tyrosine biosynthesis: confirmation in a microbial mutant. Proc Nat Acad Sci USA 1980, 77: $1270-1273$. 
44. Byng GS, Berry A, Jensen RA: Evolutionary implications of features of aromatic amino acid biosynthesis in the genus Acinetobacter. Arch Microbiol 1985, I 43: I 22-129.

45. Porat I, Waters BW, Teng Q, Whitman WB: Two biosynthetic pathways for aromatic amino acids in the archaeon Methanococcus maripaludis. J Bacteriol 2004, I 86:4940-4950.

46. Calhoun DH, Bonner CA, Gu W, Xie G, Jensen RA: The emerging periplasm-localized subclass of AroQ chorismate mutases, exemplified by those from Salmonella typhimurium and Pseudomonas aeruginosa. Genome Biol 2001:2research0030.1-0030.16.

47. Ahmad S, Jensen RA: The stable evolutionary fixation of a bifunctional tyrosine-pathway protein in enteric bacteria. Microbiol Lett 1988, 52:109-116.

48. Jensen RA, Ahmad S: Nested gene fusions as markers of phylogenetic branchpoints in prokaryotes. Trends Ecol Evol 1990, 5:219-224

49. Jensen RA, Gu W: Evolutionary recruitment of biochemically specialized subdivisions of Family I within the protein superfamily of aminotransferases. J Bacteriol 1996, 178:2161-2171.

50. Aravind L, Koonin EV: Gleaning non-trivial structural, functional and evolutionary information about proteins by iterative database searches. J Mol Biol 1999, 287: 1023-1040.

51. Henner D, Yanofsky C: Bacillus subtilis and other gram-positive bacteria. In Biochemistry, physiology, and molecular genetics Edited by: Sonenshein AL, Hoch J, Losick R. Washington, DC: ASM Press; 1993.

52. White RH: L-Aspartate semialdehyde and a 6-deoxy-5-ketohexose I-phosphate are the precursors to the aromatic amino acids in Methanocaldococcus jannashii. Biochemistry 2004 43:76|8-7627.

53. Ahmad S, Johnson JL, Jensen RA: The recent evolutionary origin of the phenylalanine-sensitive isozyme of 3-deoxy- $D$-arabinoheptulosonate 7-phosphate synthase in the enteric lineage of bacteria. J Mol Evol 1987, 25:159-167.

54. Jensen RA, Xie G, Calhoun DH, Bonner CA: The correct phylogenetic relationship of KdsA (3-deoxy-D-manno-octulosonate 8-phosphate synthase) with one of two independently evolved classes of AroA (3-deoxy-D-arabino-heptulosonate 7-phosphate synthase). J Mol Evol 2002, 54:4l6-423.

55. Pittard AJ, Camakaris H, Yang J: The TyrR regulon. Mol Microbiol 2005, 55:16-26.

56. Katayama T, Suzuki H, Koyanagi T, Kumagai $H$ : Cloning and random mutagenesis of the Erwinia herbicola tyrR gene for highlevel expression of tyrosine phenol-lyase. Appl Envir Microbiol 2000, 66:4764-4771.

57. Bai Q, Somerville R: Integration host factor and cyclic AMP receptor proein are required for TyrR-mediated activation of tpl in Citrobacter freundii. J Bacteriol 1998, 180:6173-6186.

58. Zhao S, Somerville RL: Isolated operator binding and ligand response domains of the TyrR protein of Haemophilus influenzae associate to reconstitute functional repressor. I Bio Chem 1999, 274: 1842-1847.

59. Arias-Barrau E, Olivera E, Luengo J, Fernandez C, Galan B, Garcia J, Diaz E, Miñambres B: The homogentisate pathway: a central catabolic pathway involved in the degradation of $L$-phenylalanine, L-tyrosine, and 3-hydroxyphenylacetate in Pseudomonas putida. I Bacteriol 2004, 186:5062-5077.

60. Dähnhardt D, Falk J, Appel J, van der Kooij A, Schulz-Friedrich R, Krupinska K: The hydroxyphenylpyruvate dioxygenase from Synechocystis sp. PCC $\mathbf{6 8 0 3}$ is not required for plastoquinone biosynthesis. FEBS Lett 2002, 523:177-I81.

61. Song J, Jensen RA: PhhR, a divergently transcribed activator of the phenylalanine hydroxylase gene cluster of Pseudomonas aeruginosa. Mol Microbiol 1996, 22:497-507.

62. Zhao G, Xia T, Song J, Jensen R: Pseudomonas aeruginosa possesses homologues of mammalian phenylalanine hydroxylase and 4a-carbinolamine dehydratase/DCoH as part of a three-component gene cluster. Proc Natl Acad Sci USA 1994 91:1366-1370

63. Tropel D, van der Meer J: Bacterial transcriptional regulators for degradation pathways of aromatic compounds. Microbiol Mol Biol Rev 2004, 68:474-500.

64. Chaney M, Grande R, Wigneshweraraj S, Cannon W, Casaz P, Gallegos M-T: Binding of transcriptional activators to sigma 54 in the presence of the transition state analog ADP-aluminum fluoride: insights into activator mechanochemical action. Genes Dev 200I, I 5:2282-2294.
65. Yanofsky C: The different roles of tryptophan transfer RNA in regulating trp operon expression in $E$. coli versus $B$. subtilis. Trends Genet 2004, 20:367-374.

66. Predicted attenuators in bacteria [http://cmgm.stanford.edu/ $\sim$ merino]

67. Riley ML, Schmidt T, Wagner c, Mewes H-W, Frishman D: The PEDANT genome database in 2005. Nuc Ac Res 2005, 33:D308-D310.

68. Chenna R, Sugawara H, Koike T, Lopez R, Gibson T, Higgins D, Thompson J: Multiple sequence alignment with the Clustal series of programs. Nucl Ac Res 2003, 31 I:3497-3500.

69. BioEdit [http://www.mbio.ncsu.edu/BioEdit/bioedit.html]

70. Felsenstein J: PHYLIP-Phylogeny Inference Package (version 3.2). Cladistics 1989, 5:164-166.

7I. Cai W, Pei J, Grishin NV: Reconstruction of ancestral protein sequences and its applications. BMC Evol Biol 2004, 4:33.

72. Eddy S: HMMER package. 1995 [http://hmmer.wustl.edu]

73. AroPath [http://AroPath.lanl.gov/Annotation/TyrA/ TyrA substrateSpc.html]

74. AroPath Species sorted by acronym.html [http://AroPath.lanl.gov/Organisms/
Publish with Bio Med Central and every scientist can read your work free of charge

"BioMed Central will be the most significant development for disseminating the results of biomedical research in our lifetime. "

Sir Paul Nurse, Cancer Research UK

Your research papers will be:

- available free of charge to the entire biomedical community

- peer reviewed and published immediately upon acceptance

- cited in PubMed and archived on PubMed Central

- yours - you keep the copyright
BioMedcentral 